\title{
Energy-flux relationship in the FUV Jovian aurora deduced from HST-STIS spectral observations
}

\author{
J. Gustin, ${ }^{1}$ J.-C. Gérard, ${ }^{1}$ D. Grodent, ${ }^{1}$ S. W. H. Cowley, ${ }^{2}$ J. T. Clarke, ${ }^{3}$ and A. Grard ${ }^{1}$
}

Received 22 December 2003; revised 8 June 2004; accepted 16 July 2004; published 15 October 2004.

[1] Far ultraviolet spectral observations of the Jovian aurora have been made since 1997 with the Space Telescope Imaging Spectrograph (STIS) on board the Hubble Space Telescope at low spectral resolution. The combination of the spectral resolution with the intensity variation along the STIS slit provides information on the latitudinal variation of the precipitating auroral electron energy flux and the mean electron energy, from which the electron current density at the top of the atmosphere can also be deduced. It is found that the mean electron energies associated with the main oval lie in the range $30-200 \mathrm{keV}$ and show a tendency to increase with the precipitating energy flux. The current densities lie in the range $\sim 0.04-0.4 \mu \mathrm{A} \mathrm{m}^{-2}$, consistent with previous estimates, and are also positively correlated with the energy flux. The observed relationship between the auroral time-integrated energy fluxes and the electron energies in the main oval is compatible with that expected from Knight's theory of field-aligned currents. The best agreement between the observed data and the Knight curves is obtained for an electron temperature of $\mathrm{T}_{\mathrm{e}}=2.5 \mathrm{keV}$ and a source density $\mathrm{N}=0.003 \mathrm{~cm}^{-3}$, that is within the range of values observed in the equatorial plane during the Voyager flybys. No systematic dependence of the electron energy with magnetic local time is found, but the morning sector around 0800 MLT shows greater variability than other regions of the oval. Analysis of time-tagged data shows that the main oval energy flux usually varies steadily over the several minute intervals of observation and that the mean electron energy usually undergoes correlated variations such that the current density remains relatively constant. It is shown that these overall properties are also consistent with Knight's theory of auroral electron acceleration associated with field-aligned current flow, from which it is inferred that the temporal variations observed are often due to slow changes in the magnetospheric "source" electron parameters in the presence of near-steady magnetosphere-ionosphere coupling currents. By contrast, time-integrated emissions in the polar region are found to be associated with similar mean electron energies to the main oval but with typically smaller energy fluxes and current densities. Pressure balance arguments are advanced, which indicate that the brighter of these emissions must be associated with an auroral acceleration mechanism perhaps similar to that operative in the main oval, while it remains possible that the weaker emissions could result from precipitation from a quasi-isotropic hot magnetospheric electron source. INDEX TERMS: 6220 Planetology: Solar System Objects: Jupiter; 2704 Magnetospheric Physics: Auroral phenomena (2407); 2716 Magnetospheric Physics: Energetic particles, precipitating; 7807 Space Plasma Physics: Charged particle motion and acceleration; KEYWORDS: Jovian aurora, HST/STIS spectra, field-aligned currents, energy of precipitated electrons

Citation: Gustin, J., J.-C. Gérard, D. Grodent, S. W. H. Cowley, J. T. Clarke, and A. Grard (2004), Energy-flux relationship in the FUV Jovian aurora deduced from HST-STIS spectral observations, J. Geophys. Res., 109, A10205, doi:10.1029/2003JA010365.

\footnotetext{
${ }^{1}$ Laboratoire de Physique Atmosphérique et Planétaire, Université de Liège, Liège, Belgium.

${ }^{2}$ Department of Physics and Astronomy, Leicester University, Leicester, United Kingdom. USA.

${ }^{3}$ Center for Space Physics, Boston University, Boston, Massachusetts,

Copyright 2004 by the American Geophysical Union. 0148-0227/04/2003JA010365\$09.00
}

\section{Introduction}

[2] The FUV Jovian aurora is characterized by three main regions of emission [Gérard et al., 1994; Clarke et al., 1996; Grodent et al., 2003a]. The first is the main auroral oval that is magnetically connected to the middle magnetosphere at $\sim 20-30 R_{J}\left(R_{J}=\right.$ Jupiter's radius $)$. The plasma in this region is characterized by departures from rigid corotation with the planet, which increase with the radial distance. Its morphology is characterized by a 
bright arc extending from dawn to noon or further, which may be enhanced near the dawn limb during occasional storms. In the afternoon sector, the main oval is generally more diffuse and weaker than in the morning sector. Second, diffuse emission is frequently observed in the polar region inside the main oval. It is generally weaker than the main oval but shows large time variations on time scales of minutes [Clarke et al., 1998; Gérard et al., 2003; Grodent et al., 2003b], sometimes brightening considerably in polar flares [Waite et al., 2001]. Third, we observe spots at the footprints of Io, Ganymede, and Europa [Clarke et al., 2002]. The Io footprint shows a trailing tail that sometimes extends over as much as $180^{\circ}$ of longitude [Clarke et al., 1998; Gérard et al., 2002]. Similar features have observed in infrared $\mathrm{H}_{3}^{+}$emission but will not be discussed in this study [Clarke et al., 2004]. Since the characterization of the energy of the precipitated electrons can only be achieved with ultraviolet spectra, this paper will focus on FUV observations only.

[3] In situ measurements of energetic electrons traveling along field lines connected to the Jovian aurora have been made by the Voyager, Ulysses, and Galileo spacecraft. However, they were obtained only at large distances from the ionosphere and do not necessarily indicate the characteristics of the charged particle precipitation causing the aurora. Voyager measurements of oxygen and sulfur ion distributions led Gehrels and Stone [1983] to suggest that the aurora are caused by pitch angle scattering of ions with energies of several $\mathrm{MeV} /$ nucleon at equatorial radial distances of $\sim 6$ to $17 \mathrm{R}_{\mathrm{J}}$. Field-aligned beams of electrons and ions of energies above $50 \mathrm{keV}$ were observed by the Ulysses spacecraft at radial distances from 15 to $75 \mathrm{R}_{\mathrm{J}}$ from the planet [Cowley et al., 1996; Krupp et al., 1997]. At $25 \mathrm{R}_{\mathrm{J}}$ the Ulysses spacecraft crossed magnetic field lines intercepting the Jovian ionosphere at higher latitudes than the main oval. These measurements are thus mainly associated with the highlatitude auroral emission inside the main auroral oval. Frank and Paterson [2002] presented measurements of the electron energy spectrum from 0.9 to $52 \mathrm{keV}$ obtained by the plasma instrumentation (PLS) on board the Galileo spacecraft between 9 and $50 \mathrm{R}_{\mathrm{J}}$ in the morning and premidnight magnetosphere. Intense electron beams narrowly collimated parallel and antiparallel to the magnetic field were measured at distances of $\sim 20$ to $30 \mathrm{R}_{\mathrm{J}}$ and identified with the main auroral oval. Their energies ranged from several $\mathrm{keV}$ to tens of $\mathrm{keV}$, and the energy flux they carried extended up to $\sim 100 \mathrm{~mW} \mathrm{~m}^{-2}$. The presence of intense antiparallel beams near the equatorial plane is not predicted by the model of electron acceleration by parallel electric fields in regions of upward fieldaligned currents, as pictured by Cowley and Bunce [2001] and Hill [2001] and discussed below. Frank and Peterson [2002] attributed the source of the electron beams to intense plasma heating at low altitudes. Measurements of the electron energy and pitch angle distribution made with the Energetic Particle Detector on Galileo have been used to examine whether the radiation belt population can provide the energy flux that powers the Jovian aurora. Bhattacharya et al. [2001] showed that the pitch angle distribution of electrons with energies from 15 to $884 \mathrm{keV}$ indicates the presence of an enhanced population in the loss cone over the middle magnetosphere (between 10 and $25 \mathrm{R}_{\mathrm{J}}$ ). However the energy flux associated with these precipitated electrons is less than $10 \mathrm{~mW} \mathrm{~m}^{-2}$ outside of $25 \mathrm{R}_{\mathrm{J}}$, a value that is too low to explain the bright arc-like structures in the main oval. Values in excess of $10 \mathrm{~mW} \mathrm{~m}^{-2}$ may occur over an extended region (between 10 and $25 R_{J}$ ) following strong diffusion scattering and explain the diffuse auroral emission frequently observed both in the ultraviolet and the infrared equatorward of the Jovian main auroral oval. The maximum available electron energy flux drops to values less than $1 \mathrm{~mW} \mathrm{~m}^{-2}$ outside $30 \mathrm{R}_{\mathrm{J}}$. The energy flux associated with proton precipitation is found typically to lie well below $10 \%$ of the electron flux, rarely exceeding $2 \mathrm{~mW} \mathrm{~m}^{-2}$. Bhattacharya et al. [2001] concluded that an additional acceleration process such as potential drops along the field lines associated with field-aligned currents are required to account for the intense auroral structures.

[4] FUV imaging of the polar regions with the Space Telescope Imaging Spectrograph (STIS) provides a global view of the Jovian aurora with unprecedented spatial and temporal [Clarke et al., 2002; Waite et al., 2001; Grodent et al., 2003a, 2003b] and spectral [Gustin et al., 2002; Gérard et al., 2002, 2003] resolution. Grodent et al. [2003a] examined in detail the morphology and intensity of the main oval in an extended series of FUV images obtained with the HST during winter 2000-2001. They showed that the brightness of the main oval varies from 50 to $500 \mathrm{kR}$, once reaching over $1 \mathrm{MR}$. The main oval is typically found to emit $\sim 60 \%$ of the total auroral brightness [Grodent et al., 2003b]. They also found that variations on time scales of minutes or less are much more frequent poleward of the main oval than along the oval.

[5] High-latitude spatially structured FUV emissions are frequently observed poleward of the main oval, especially in the noon and afternoon sectors of the planet. Their location and brightness can vary rapidly $(\sim 100 \mathrm{~s})$, as evidenced by changes observed in HST images taken minutes apart [Clarke et al., 1998; Grodent et al., 2003b]. Very dynamical and intense high-latitude emissions ("polar flares") were reported by Waite et al. [2001]. Gérard et al. [2003] analyzed the relationship between the precipitated electron flux and the electron mean energy remotely determined from the $\mathrm{H}_{2}$ auroral spectrum of polar transient features. They found that the temporal variations of the precipitated flux in high-latitude spots are generally not caused by changes in mean electron energy but mainly by changes in total energy flux at nearly fixed energy. By contrast, they presented a case of brightening of the main oval concurrent with an increase of the mean electron energy from 40 to $80 \mathrm{keV}$.

[6] The main oval maps magnetically to distances outside Ganymede's orbit (15 $\left.\mathrm{R}_{\mathrm{J}}\right)$ [Clarke et al., 2002], a region where the flow of plasma increasingly departs from rigid corotation [Hill, 1980; Mauk et al., 2002]. Hill [1979] showed that such departures would lead to the formation of a large-scale magnetosphere-ionosphere coupling current system, in which radial currents in the equatorial plasma sheet close in the ionosphere via field-aligned currents. Hill [2001] and Bunce and Cowley 
[2001] proposed that the main oval is related to the region of upward field-aligned current and downward precipitating electrons associated with this system. Links between field-aligned currents and auroras in a variety of Jovian physical scenarios had also been suggested in earlier work [Kennel and Coroniti, 1975; Barbosa et al., 1981; Isbell et al., 1984]. A realistic model of the corotation-related currents was developed by Cowley and Bunce [2001], which employed Knight's [1973] kinetic theory to show that large field-aligned potential drops of $\sim 50-150 \mathrm{kV}$ are required to drive the upward currents. Another auroral electron acceleration mechanism suggested by Saur et al. [2003] is based on the weak MHD turbulence observed in the middle magnetosphere plasma sheet by the Galileo magnetometer. In this picture the field-aligned potential drop necessary to accelerate the electrons originates from the coupling of the weak turbulence with Hill's [1979] large-scale current system. The power of the turbulent fluctuations maximizes between 18 and $30 \mathrm{R}_{\mathrm{J}}$, that is, on magnetic field lines threading the main auroral oval. Saur et al. [2003] estimate that a potential of $\sim 160 \mathrm{kV}$ is generated through this process and that the accelerated electrons carry a total power $\sim 6 \times 10^{12} \mathrm{~W}$ when mapped to the ionosphere. The energy flux associated with the region of maximum turbulent power corresponds to an energy flux of $\sim 7 \mathrm{~mW} \mathrm{~m}^{-2}$ at the footprint of the $20 \mathrm{R}_{\mathrm{J}}$ field line.

[7] STIS-HST spatially resolved spectra make it possible to simultaneously measure both the auroral brightness and the FUV color ratio of auroral emissions [Gustin et al., 2002]. The latter is given by

$$
C R=\frac{I(1550-1620 \AA)}{I(1230-1300 \AA)},
$$

and measures the attenuation of the $\mathrm{H}_{2}$ emission below $1350 \AA$ by hydrocarbons, dominated by methane. It was originally introduced in a slightly different form by Yung et al. [1982]. In this paper the "unabsorbed intensity" thus refers to the $1550-1620 \AA$ spectral window, the "absorbed intensity" refers to the $1230-1300 \AA$ spectral window, and the "color ratio" (CR) gives the ratio between them. The methane column density associated with the CR has been found to vary with place and time, suggesting that the characteristic electron energy is also variable. Color ratio variations may also reflect local changes in the amount of hydrocarbons near the homopause [Livengood et al., 1990], possibly induced by enhanced vertical turbulent transport. The effect of a doubling of the eddy diffusion coefficient on the determination of the mean electron energy was discussed by Gérard et al. [2003].

[8] In this study we investigate the variations of the CR and the associated characteristic electron energy which are observed in the main oval and the polar region and use them to determine the current density associated with the precipitating electrons. We examine the STIS database to identify the possible dependence of the mean auroral electron energy on local time and to determine if precipitation poleward of the oval exhibits similar characteristics to the main oval. We also compare the relationship between the electron energy, current density, and energy flux observed in the main oval and in the polar region with theoretical expectations.

\section{Observations and Data Reduction}

\subsection{Time-Integrated Color Ratio}

[9] A total of 102 FUV spectra of the north and south Jovian aurora were obtained with STIS between July 1997 and January 2001. Among these observations, 66 spectra were obtained with the G140L, 16 with the G140M, and 20 with the Echelle gratings. The moving target mode was used to acquire the spectra, implying that the aperture was placed at a fixed location with respect to the planet, and did not track a preset planetary longitude. In this study we analyze spectra obtained with the G140L grating, combined with the $25 \times 0.2 \operatorname{arcsec}^{2}$ and the $25 \times 0.5 \operatorname{arcsec}^{2}$ slits, providing spectra in the range 1150 to $1750 \AA$, with $\sim 4.8 \AA$ and $\sim 12$ A resolution, respectively. As indicated above, the 1150-1750 A spectral range includes both unabsorbed and absorbed $\mathrm{H}_{2}$ FUV emissions [see Gérard et al., 2003, Figure 1], which is ideal to derive color ratios. All the observations were made in the "time-tag" mode, without additional filters. Processing of the STIS spectra and background subtraction were described by Gustin et al. [2002] and can be summarized as follows.

[10] We use the spectroscopic "flat-fielded" ( flt $)$ science files, which provide time-integrated spectra in the dispersion direction and a uniform sampling in the spatial direction. It is known from the previous work of Landsman [1998] and Gustin et al. [2002] that the dark signal (the count rate in the detector in the absence of photons from the sky) can be very inhomogeneous in the $1024 \times 1024$ pixel MAMA detector and may produce an extra signal, which is concentrated in the upper left portion of the detector. The subtraction of this time-variable "blotch" is not taken into account by the automatic pipeline procedure. Consequently, each observation was processed using the best estimate of the dark count level available. We note that the "dark blotch" is not as important for the G140L observations used here, compared with the G140M observations described by Gustin et al. [2002]. This is due to the higher count rate of the G140L spectra and the position of the aurora on the MAMA detector relative to the "blotch" region.

[11] The second processing step is to extract from the MAMA detector the auroral signal itself. Gustin et al. [2002, Figure 1] shows an example of the STIS intensity distribution along the slit, which reflects the auroral morphology. In order to obtain a reliable database of the main oval and polar cap color ratios, we only used spectra that were obtained close in time (before or after) a STIS image, in order to clearly identify the features seen in the twodimensional (2-D) spectrum. To locate the slit of a given spectrum on the related image, the method described by Gustin et al. [2002] is used. A number of columns of the image corresponding to the width of the slit are summed, and the resulting intensity curve is compared with the intensity obtained along the slit by integrating the spectrum. The location of the slit on the image is then displaced until a good match is obtained. An example of such curves can be found in Figure 5 of Gustin et al. [2002]. The accuracy of this method is estimated to be approximately 5 pixels 

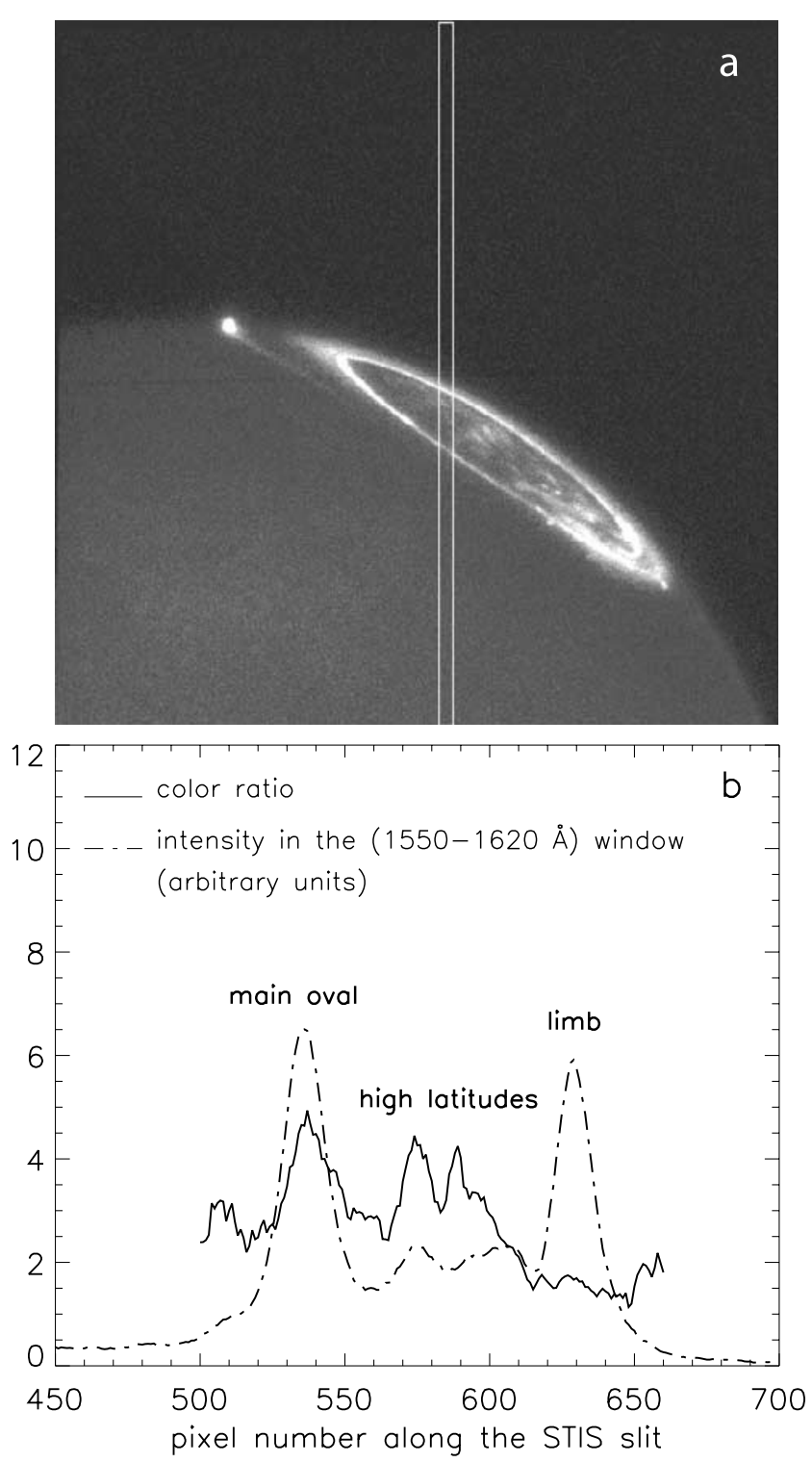

Figure 1. (a) Image obtained at 0217 UT on 26 November 1998 (case 6 in Table 1) with the STIS MAMA aperture. The main regions of the Jovian aurora consisting of limb aurora, high-latitude emission, main oval, and Io tail are easily identified. The position of the STIS slit used to obtain spatially resolved spectra of the Jovian aurora is marked by the double line crossing the polar region. (b) Variation along the STIS slit (from bottom to top) of the unabsorbed $\mathrm{H}_{2}$ intensity (dot-dashed line) and the color ratio (solid line). Each auroral region is characterized by a different CR.

$(0.1$ arcsec), assuming that the auroral morphology does not change significantly between the two measurements.

[12] In addition to these steps, a further procedure was needed to obtain the final data analyzed here. Some of the G140L spectra were obtained when HST was observing through the Earth's sunlit exosphere and thus include the bright atomic oxygen dayglow triplet at $1304 \AA$. These spectra can easily be identified, as the $1304 \AA$ feature evenly fills the STIS aperture. Because of the low resolution of the G140L grating mode, this oxygen feature may contribute to the $1230-1300 \AA$ spectral window and corrupt the derived values of the CR. All spectra including the $1304 \AA$ oxygen feature were thus rejected from our spectral database. A total of 23 spectra were finally used in this study. The characteristics of the exposures are listed in Table 1. To calculate the CR, the flt files, which are in units of counts/pixel, need to be corrected for the detector sensitivity. After correction, each line of the flt file provides a spectrum in units of $\mathrm{mW} \mathrm{m} \mathrm{m}^{-2} \AA^{-1} \operatorname{arcsec}^{-2}$. This spectrum is converted into photon units (Rayleighs (R) $\AA^{-1}$ ), and the ratio between the $1550-1620 \AA$ and the 1230-1300 $\AA$ regions determines the CR. This ratio is calculated for each line of the flt file, such that it is possible to examine the variation along the slit of the CR. For this part of the study, a single value of the CR is determined to characterize each region of the aurora. Before STIS was installed on board HST in 1997, it was not possible to discriminate between the different regions of aurora. Observations made by Voyager UVS, IUE, HUT, HST GHRS, and Galileo EUVS did not provide detailed spatially resolved spectra. For this reason the CRs derived with these instruments were coarse compared with the spatially resolved data provided by STIS. Figure 1a shows an example of a FUV image of the Jovian north polar region taken a few minutes before a STIS spectrum. As seen in Figure $1 \mathrm{~b}$, the CR for, say, the main oval, can be defined in different ways. The $\mathrm{CR}$ at the peak of the unabsorbed intensity reaches 5.2 , while the mean value of the CR from pixel number 525 to 548 (the visually determined spatial range used to define the main oval) is 3.9. This means that the $\mathrm{CR}$ is dependent on the spatial range used to define the auroral region which is to be analyzed. To minimize the effect of the choice of the main oval boundary, the CR in the selected spatial window is weighted by the unabsorbed intensity in the window. That is, we use the weighted value

$$
C R=\frac{\sum_{x=a}^{b}\left[I_{\text {ua }}(x) * C R(x)\right]}{\sum_{x=a}^{b} I_{u a}(x)},
$$

where $I_{u a}(x)$ is the unabsorbed intensity of the selected auroral region for pixel number $x$ and $C R(x)$ is the corresponding CR. Taking Figure $1 \mathrm{~b}$ as an example, the weighted CR of the main oval is calculated with $a=525$ and $b=548$, giving a weighted $\mathrm{CR}$ value of 4.1. This procedure was used to derive the CRs employed in this study.

\subsection{Time-Dependent Color Ratio}

[13] Time variations of the main oval and polar emissions in the 23 spectra studied here were also examined, using information provided by the time-tag (tag) files. Timetagged data are stored in an event table, containing separate columns for the pixel coordinates and the arrival time of each detected photon event, with an integration period of $125 \mu \mathrm{s}$. To achieve a good signal-to-noise $(\mathrm{S} / \mathrm{N})$ ratio while keeping a sufficient time resolution, the photon events were integrated over $10 \mathrm{~s}$ intervals, giving a $\mathrm{S} / \mathrm{N}$ ratio in the order of $\sim 8$. Photon events in the 1230-1300 $\AA$ and 1550$1620 \AA$ spectral windows were summed over $10 \mathrm{~s}$ to derive 
Table 1. Dates and Characteristics of STIS Observations Used in This Study ${ }^{\mathrm{a}}$

\begin{tabular}{|c|c|c|c|c|c|c|c|c|c|c|}
\hline Obs & Date & $\begin{array}{c}\text { Start } \\
\text { Time, } \\
\text { UT }\end{array}$ & $\begin{array}{c}\text { Exposure, } \\
\mathrm{S} \\
\end{array}$ & $\begin{array}{l}\text { CML } \\
\left(\lambda_{\text {III }}\right), \\
\text { deg }^{\mathrm{b}}\end{array}$ & $\begin{array}{l}\text { Local } \\
\text { Time }\end{array}$ & $\mathrm{N} / \mathrm{S}$ & $\begin{array}{l}\text { MO Mean } \\
\text { Intensity, } \\
\mathrm{kR}^{\mathrm{c}} \\
\end{array}$ & $\mathrm{CR}^{\mathrm{d}}$ & $\begin{array}{c}\text { Relative } \\
\text { Intensity Time } \\
\text { Variation }^{\mathrm{e}} \\
\end{array}$ & $\begin{array}{c}\text { Relative CR } \\
\text { Time } \\
\text { Variation } \\
\end{array}$ \\
\hline 1 & 04 Jul 97 & 0931 & 700 & 52.5 & - & $\mathrm{S}$ & 252 & 3.6 & $2.0 \rightarrow 1.0$ & cst \\
\hline 2 & 04 Jul 97 & 0947 & 1400 & 62.8 & - & $\mathrm{S}$ & 173 & 2.4 & $1.0 \rightarrow 2.9$ & $1.7 \rightarrow 1.0$ \\
\hline 3 & 20 Sep 97 & 1436 & 400 & 107.5 & $\mathrm{AM}$ & $\mathrm{S}$ & 75 & 4.9 & cst & cst \\
\hline 4 & 20 Sep 97 & 1613 & 400 & 166.1 & PM & $\mathrm{N}$ & 313 & 3.1 & cst & $1.0 \rightarrow 1.7$ \\
\hline 5 & 26 Jul 98 & 1401 & 920 & 116.5 & PM & $\mathrm{N}$ & 99 & 1.8 & $1.2 \rightarrow 1.0$ & cst \\
\hline 6 & 26 Nov 98 & 0217 & 600 & 214.8 & PM & $\mathrm{N}$ & 57 & 4.1 & cst & cst \\
\hline 7 & 08 Aug 99 & 1310 & 200 & 61.5 & $\mathrm{AM}$ & $\mathrm{S}$ & 23 & 2.1 & cst & cst \\
\hline 8 & 08 Aug 99 & 1317 & 200 & 61.5 & AM & $\mathrm{S}$ & 24 & 1.8 & cst & cst \\
\hline 9 & $22 \mathrm{Feb} 00$ & 1647 & 240 & 127.3 & $\mathrm{AM}$ & $\mathrm{N}$ & 83 & 9.6 & $1.0 \rightarrow 1.6$ & $1.0 \rightarrow 1.7$ \\
\hline \multirow[t]{2}{*}{10} & 14 Nov 00 & 0606 & 230 & 151.8 & AM & $\mathrm{N}$ & 193 & 8.3 & $1.0 \rightarrow 3.7$ & $1.0 \rightarrow 1.6$ \\
\hline & & & & & & & 304 & 4.5 & $1.0 \rightarrow 2.0$ & $1.0 \rightarrow 1.3$ \\
\hline \multirow[t]{2}{*}{11} & 14 Nov 00 & 0614 & 230 & 156.6 & AM & $\mathrm{N}$ & 279 & 8.2 & $1.0 \rightarrow 2.4$ & $1.0 \rightarrow 1.6$ \\
\hline & & & & & & & 293 & 4.2 & $1.3 \rightarrow 1.0$ & cst \\
\hline \multirow[t]{2}{*}{12} & 14 Nov 00 & 0728 & 230 & 201.4 & $\mathrm{AM}$ & $\mathrm{N}$ & 62 & 2.5 & $1.0 \rightarrow 1.5$ & 2.4. $\rightarrow 1.0$ \\
\hline & & & & & & & 50 & 1.5 & cst & cst \\
\hline \multirow[t]{2}{*}{13} & 14 Nov 00 & 0736 & 270 & 206.2 & AM & $\mathrm{N}$ & 87 & 2.5 & $1.6 \rightarrow 1.0$ & cst \\
\hline & & & & & & & 27 & 1.9 & cst & cst \\
\hline 14 & 14 Nov 00 & 0744 & 270 & 211.9 & $\mathrm{AM}$ & $\mathrm{N}$ & 130 & 2.7 & cst & cst \\
\hline 15 & 14 Dec 00 & 1221 & 630 & 218.5 & PM & $\mathrm{N}$ & 36 & 2.4 & $1 \rightarrow 1.3$ & $1.0 \rightarrow 1.3$ \\
\hline 16 & 14 Dec 00 & 1237 & 630 & 228.1 & PM & $\mathrm{N}$ & 198 & 3.8 & $1.5 \rightarrow 1.0$ & cst \\
\hline \multirow[t]{2}{*}{17} & 16 Dec. 00 & 1234 & 630 & 168.0 & PM & $\mathrm{N}$ & 56 & 4.5 & $8.0 \rightarrow 1.0$ & $4.5 \rightarrow 1.0$ \\
\hline & & & & & & & 40 & 3.8 & $3.3-1.0$ & $3.5-1.0$ \\
\hline 18 & 16 Dec. 00 & 1251 & 630 & 177.2 & PM & $\mathrm{N}$ & 87 & 3.1 & $2.4 \rightarrow 1.0$ & cst \\
\hline 19 & 20 Jan 01 & 1558 & 260 & 160.3 & AM & $\mathrm{N}$ & 23 & 2.2 & cst & cst \\
\hline 20 & 20 Jan 01 & 1608 & 260 & 166.4 & PM & $\mathrm{N}$ & 118 & 4.5 & cst & cst \\
\hline 21 & 21 Jan 01 & 2229 & 260 & 187.2 & $\mathrm{AM}$ & $\mathrm{N}$ & 126 & 3.2 & cst & cst \\
\hline \multirow[t]{2}{*}{22} & 21 Jan 01 & 2239 & 260 & 193.2 & AM & $\mathrm{N}$ & 164 & 5.7 & $1.3 \rightarrow 1.0$ & $1.4 \rightarrow 1.0$ \\
\hline & & & & & & & 132 & 5.6 & $1.4 \rightarrow 1.0$ & $1.7 \rightarrow 1.0$ \\
\hline 23 & 21 Jan 01 & 2249 & 260 & 200.1 & AM & $\mathrm{N}$ & 26 & 2.0 & $1.0 \rightarrow 1.7$ & $1.0 \rightarrow 1.4$ \\
\hline
\end{tabular}

${ }^{\text {a }}$ Splitting of rows $10,11,12,13,17$, and 22 stands for cases where the main oval separates into two arcs.

${ }^{\mathrm{b}} \mathrm{CML}$ at the start time of observation.

${ }^{\mathrm{c}}$ Total $\mathrm{H}_{2}$ unabsorbed intensity in the $[1140-1700 \AA$ ] window, converted to vertical value.

${ }^{\mathrm{d}}$ Mean value, weighted by the intensity.

${ }^{\mathrm{e}}$ Variation from start to end of the exposure, except observations 2 and 17.

the CR, while the more extended unabsorbed 1500-1700 $\AA$ range was used to determine the total unabsorbed $\mathrm{H}_{2}$ intensity in the 1140-1700 $\AA$ spectral window [Gérard et al., 2003]. The last two columns of Table 1 describe the global variations of the intensities and color ratios during the observation intervals. Comparisons between time evolution of the unabsorbed intensities and the color ratios will be discussed in the next section, as well as a detailed study of some typical cases.

\section{Analysis}

\subsection{Time-Integrated Color Ratio}

\subsubsection{Variation Along the Slit}

[14] As indicated above, the spatial resolution of STIS can be used to examine the variation of the CR along the slit. Figures 1 and 2 show two examples, corresponding to cases 6 and 23 in Table 1, respectively. In these figures the various auroral regions are clearly identified in both the image and the spectrum along the slit. In this example, the shape of the CR for the main oval follows the variation of the unabsorbed intensity. Since the CR is a measure of the methane absorption in the auroral atmosphere, its values outside the region of auroral emission are irrelevant and are thus not shown. Similar shapes in the CR and intensity profiles of the main oval are observed in 21 cases out of our set of 29 (23 observations plus 6 cases where the main oval splits into two separate ovals). In the remaining eight cases the $\mathrm{CR}$ is found not to vary with the intensity. An example is shown in Figure 2, where the CR remains constant along the slit, independent of the pixel position within the aurora.

[15] Occasionally, the main oval is observed to split into more than one component, as described previously by Grodent et al. [2003a]. One such case where the main oval separates into two distinct components during the $230 \mathrm{~s}$ acquisition of the spectrum is shown in Figure 3. The spectrum was obtained starting at 0606 UT on 14 November 2000 (case 10 in Table 1), while the image was obtained 8 min before, starting at 0558 UT. The intensity distribution of the main oval region at absorbed wavelengths below $1300 \AA$ reveals only one arc, while the unabsorbed intensity above $1550 \AA$ shows two distinct regions along the slit (Figures $3 \mathrm{~b}$ and $3 \mathrm{c}$ ). This structure is also clearly seen in the FUV image, where the main oval near the STIS slit divides into two separate bright arcs. The unfiltered MAMA mode used to obtain the images in Figures $1-3$ is sensitive from 1150 to $1750 \AA$, with a maximum efficiency near $1500 \AA$. Consequently, the auroral emission observed in the images is very similar to that obtained with the G140L spectra. The distribution of intensity along the slit over the full wavelength range of the spectra is directly comparable to that of the related image and was used to superimpose the slit on the images as described above. In the case shown in Figure 3, the total intensity of the spectrum is dominated by the unabsorbed component, such that the unabsorbed 

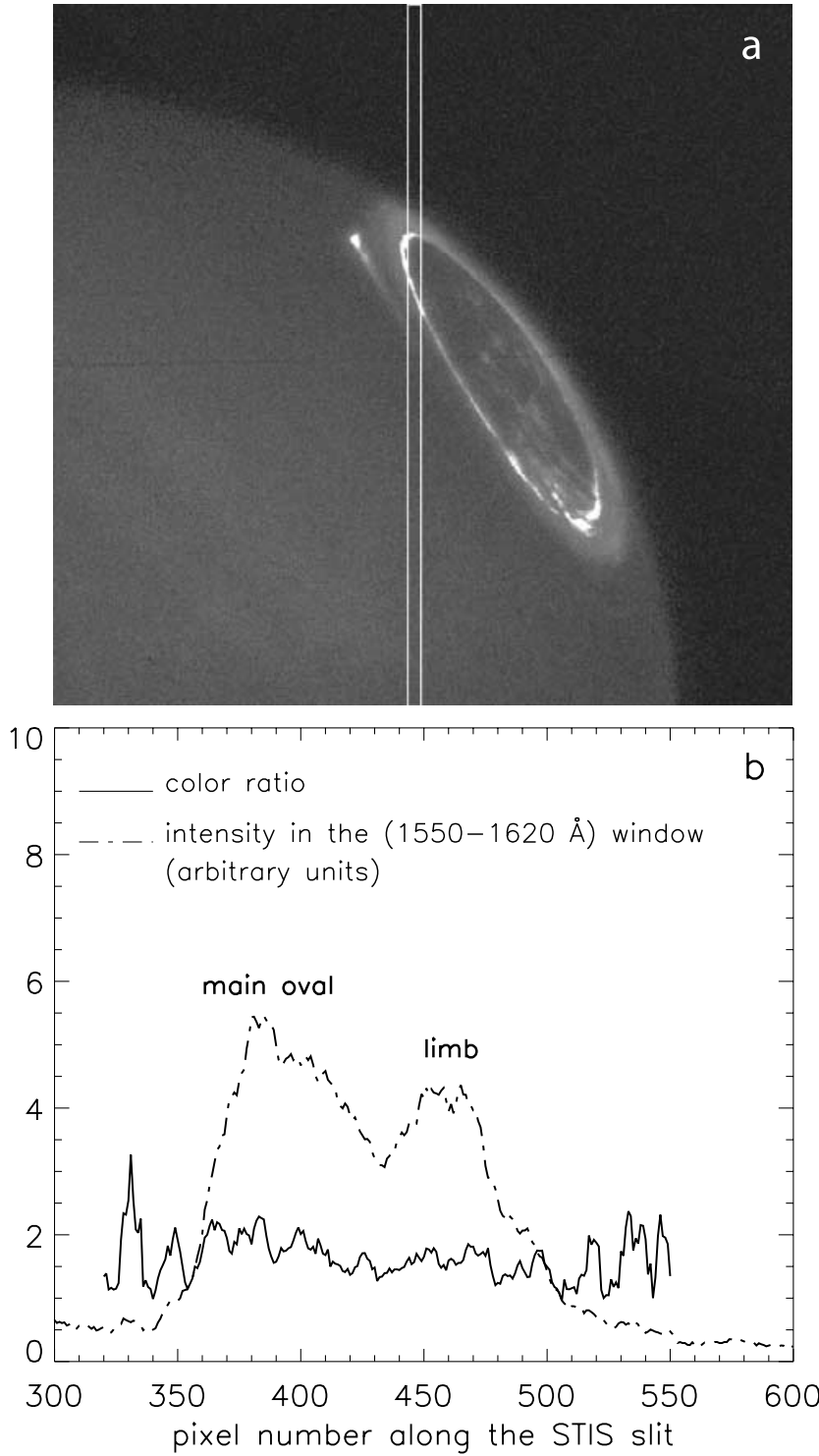

Figure 2. As for Figure 1 except for case 23 in Table 1, for which the image was obtained at 2301 UT on 21 January 2001. In this case the $\mathrm{CR}$ value is independent of the auroral region observed by the slit.

intensity shown in Figure $3 \mathrm{~b}$ is quite similar to the auroral morphology displayed in the image in Figure 3a. A consequence of the different intensity distribution observed for the absorbed and unabsorbed regions is that the color ratios for the two subovals are significantly different, in terms of their mean value and their variation along the slit, as seen in Figure 3c. The weighted CR associated with the oval at higher latitude is 8.3 , and its peak is shifted toward the limb when compared with the position of the peak in the unabsorbed intensity, while the CR at somewhat lower latitudes is 4.5 . Such latitudinal shifts are seen in 15 out of the 21 cases, where the unabsorbed intensity and CR profiles have a comparable shape along the slit. This result indicates that the two components of the oval, although relatively close in space, correspond to precipitation of electrons with different mean energies. Their origin is unknown but is has been suggested by Grodent et al. [2003a] that they are produced by a stepwise breakdown in corotation of the equatorial plasma.

\subsubsection{Mean Electron Energy-Energy Flux Relationship}

[16] As mentioned before, the $\mathrm{CR}$ is a measure of the absorption of the $\mathrm{H}_{2}$ auroral emission by hydrocarbons located within or above the region of auroral energy
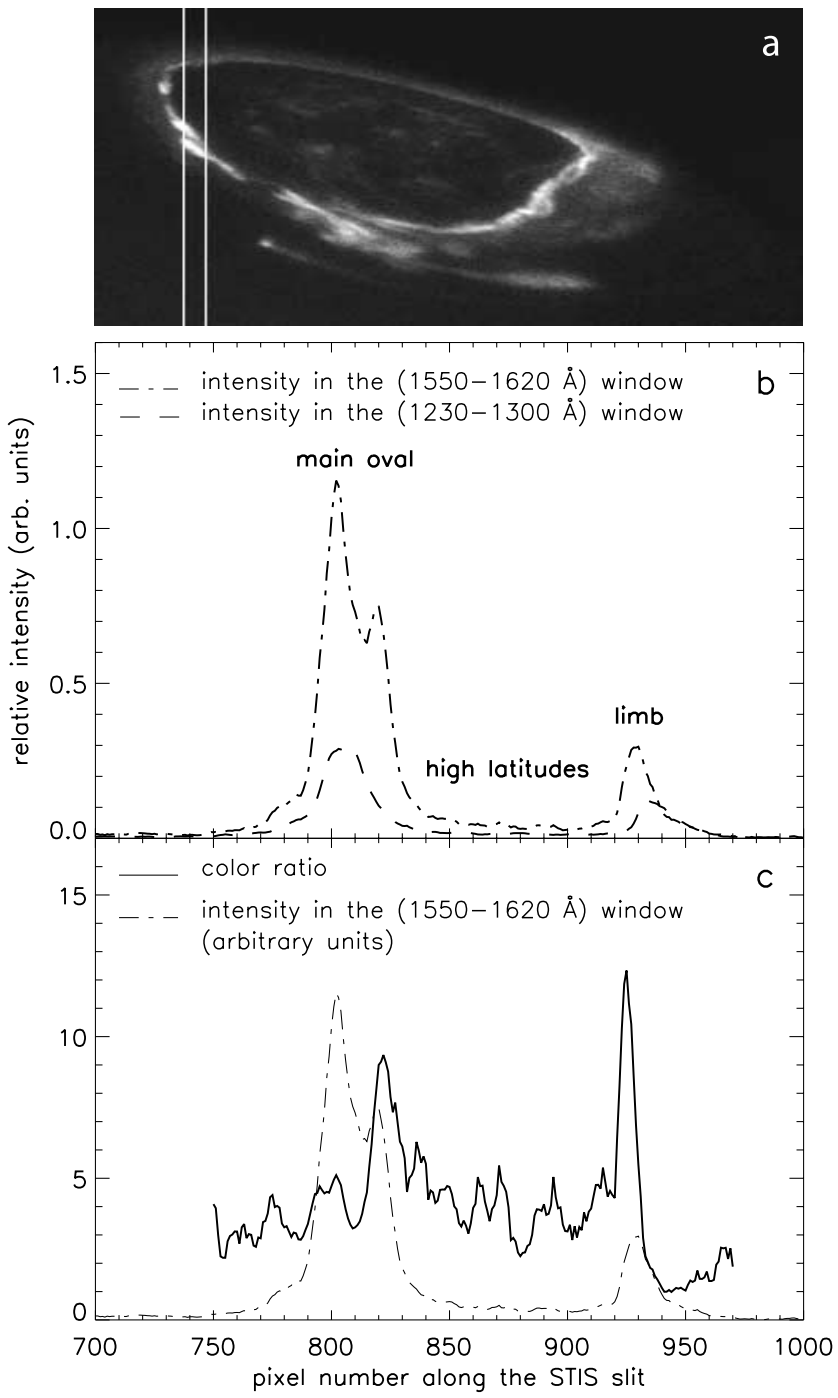

Figure 3. (a) Same as Figure 1a but for the STIS image obtained at 0606 UT on 14 November 2000 (case 10 in Table 1); the image has also been zoomed in this presentation. (b) Comparison between the absorbed (dashed line) and unabsorbed (dot-dashed line) $\mathrm{H}_{2}$ intensity along the slit. The emission in the unabsorbed $1550-1620 \AA$ spectral window clearly shows two structures in the main oval region, which is not visible in the absorbed $1230-1300 \AA$ spectral window. (c) Variation along the slit of the unabsorbed intensity, compared with the color ratio. Because of the very different profiles of the absorbed and unabsorbed intensities, the CR profile shows two peaks which are shifted toward and away from the limb compared with the peaks of the unabsorbed intensity. 

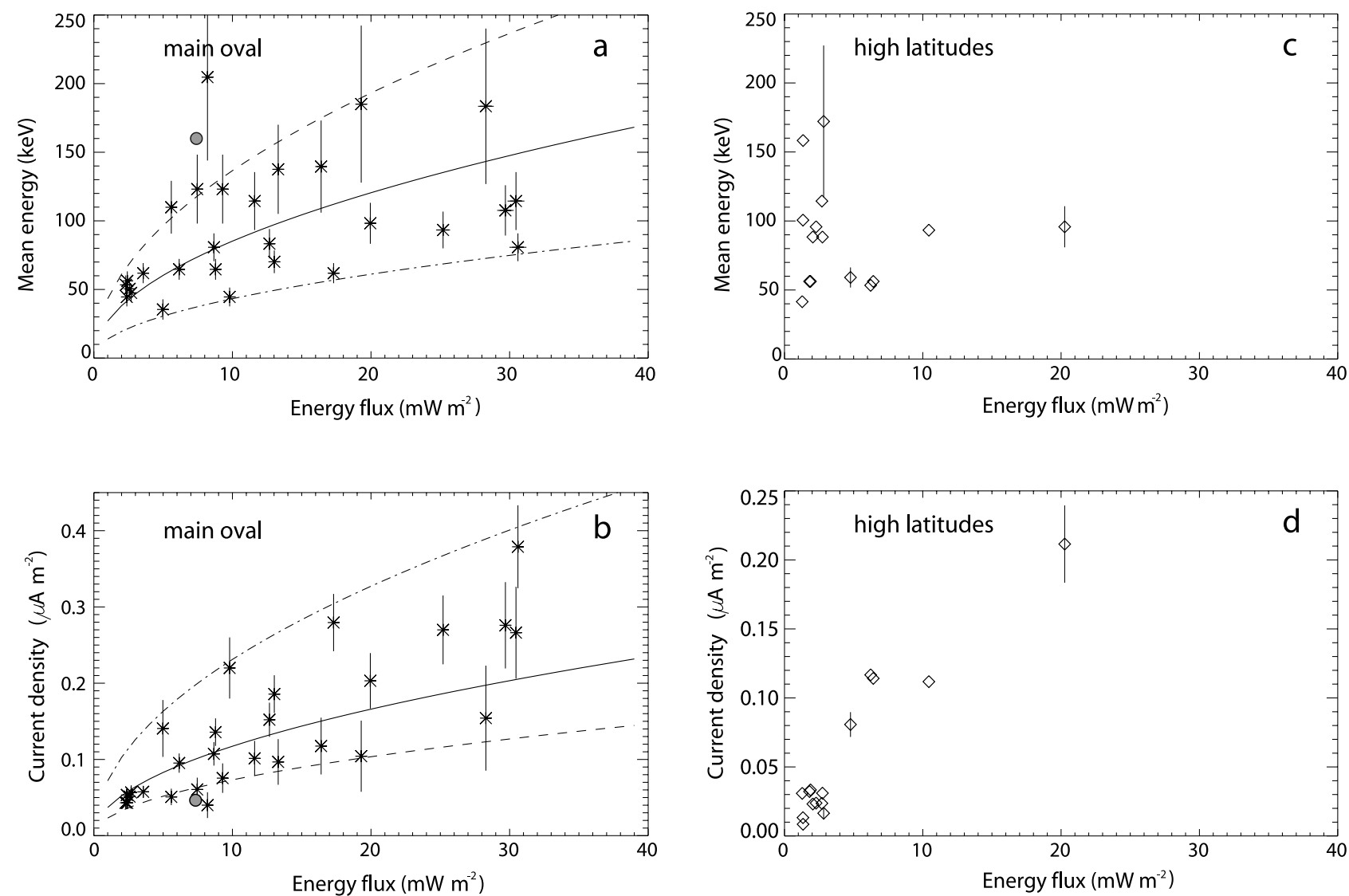

Figure 4. (a) Relationship between the mean energy of the precipitating electrons and the electron energy flux for the main oval observations. Double ovals are displayed as two distinct stars. Theoretical curves based on Knight's [1973] kinetic theory of field-aligned currents are compared with the observations. A best fit is obtained with $W_{t h}=2.5 \mathrm{keV}$ and $N=0.0026 \mathrm{~cm}^{-3}$ (central solid line). Fixing $W_{t h}$ at $2.5 \mathrm{keV}$, it is seen that the data points are bracketed by curves with $N=0.001 \mathrm{~cm}^{-3}$ (dashed line) and $N=0.01 \mathrm{~cm}^{-3}$ (dot-dashed line). The large dot stands for an estimate based on Saur et al. [2003] (b) Shows the corresponding electron current density plotted versus electron energy flux for the main oval observations. Theoretical curves based on Knight's [1973] theory are similarly superposed using the same values and line types as in Figure 4a. (c) Same as Figure 4a but for high-latitude observations. The electron flux in the polar regions is significantly lower than for the main oval. (d) Same as Figure 4c except for the high-latitude observations.

deposition. It is thus related to the optical depth of the auroral peak and hence provides an indirect measure of the energy of the precipitating electrons. Assuming a Maxwellian distribution for the primary electrons at the top of the atmosphere and a fixed value of the eddy diffusion coefficient at the homopause $K_{h}=1.4 \times$ $10^{6} \mathrm{~cm}^{2} \mathrm{~s}^{-1}$ (thus neglecting possible composition changes induced by the auroral energy input), one can derive the mean energy of the precipitating electrons using an atmospheric model of the Jovian atmosphere [Grodent et al., 2001]. The relationship between the FUV color ratio and the electron mean energy depends on the altitude distribution of the hydrocarbons. This relationship was illustrated in Figure 4 of Gérard et al. [2002] for the standard model atmosphere of Gladstone et al. [1996] and in Figure 9 of Grodent et al. [2001] for a thermally adjusted model. However, the value of the eddy diffusion coefficient may be increased in regions of enhanced particle precipitation and heating, as discussed by Livengood et al. [1990]. If the eddy diffusion is enhanced in regions of continuous auroral precipitation such as the main oval, the color ratio-energy relationship becomes modified such that the electron energy associated with a given CR is lowered [Gérard et al., 2003]. A detailed calculation of these effects would require knowledge of the three-dimensional time-dependent distribution of auroral energy input and is beyond the scope of this study. Here we have therefore made a rough estimate of the uncertainties introduced by considering the effect of doubling the eddy diffusion coefficient given above.

[17] Simulations show that for a given precipitated auroral energy spectrum, the observed color ratio also depends moderately on the viewing angle [Gérard et al., 1994]. For example, for a precipitating Maxwellian population with $\mathrm{E}_{0}=20 \mathrm{keV}$, the color ratio increases from 1.7 at normal incidence to 2.1 at $80^{\circ}$ and to 2.5 for a tangent view. For this reason, limb observations have not been used in this study. For the observations which are employed here the view angle does not exceed $80^{\circ}$, such that we have not corrected the CR data for this effect. 
[18] The stars in Figure 4a show the relationship between the mean energy of the precipitating electrons at the top of the atmosphere and the electron energy flux for all the measurements made in the main oval region (the lines and solid dot show comparisons with theoretical estimates to be discussed below.) The energy flux value assumes that an electron energy flux of $1 \mathrm{~mW} \mathrm{~m} \mathrm{~m}^{-2}$ produces $\sim 10 \pm 0.2 \mathrm{kR}$ of total Lyman and Werner band emission [Gérard and Singh, 1982; Waite et al., 1983]. The uncertainty in these values associated with statistical noise in the count rate is very small. Total counts exceed 1000 for each data point, causing statistical errors below $3 \%$. Uncertainties in the mean electron energies are much larger, however, stemming from the uncertainty in the relationship with the $\mathrm{CR}$ discussed above. Simulations with a twofold increase in the eddy diffusion coefficient near the homopause indicate that the mean electron energy can vary by up to $40 \%$ when the mean energies are large, as indicated by the error bars in the figure. Nevertheless, the observed scatter of the mean energy values is still larger than the estimated errors and thus reflects real variability. Despite these uncertainties and the natural variability, Figure 4a clearly demonstrates that for the main oval auroras the mean energy of the precipitating electrons increases with the electron energy flux.

[19] Our results also allow us to determine a reasonably direct measure of the field-aligned current density which is carried by the precipitating electrons. Dividing the electron energy flux by the mean electron energy yields the electron number flux, and multiplication by the electron charge then yields the electron current density. This is shown for the main oval versus the energy flux in Figure 4b. It can be seen that the implied current densities range typically between $\sim 0.04$ and $\sim 0.4 \mu \mathrm{A} \mathrm{m}^{-2}$, thus being entirely comparable with those deduced previously both from magnetospheric magnetic data [Bunce and Cowley, 2001; Khurana, 2001] and from theoretical models [e.g., Cowley and Bunce, 2001; Cowley et al., 2002, 2003]. Despite the scatter in the values, Figure $4 \mathrm{~b}$ also demonstrates that the current density and electron energy flux are also positively correlated quantities.

[20] The relationship between the mean energy and the energy flux can also be examined for the high-latitude regions. Figure $4 \mathrm{c}$ shows results obtained from averages over the polar region between the dayside main oval and the nightside limb emission. The smaller number of data points compared with Figure 4a results from the fact that the polar region is not always clearly observed separately from the main oval, as for example in Figure 2. The results are somewhat different from the main oval. A few points with higher energy flux $\sim 5-20 \mathrm{~mW} \mathrm{~m}^{-2}$ (thus associated with relatively bright polar emissions $\sim 50-200 \mathrm{kR}$ ) have values which are not dissimilar to the main oval distribution. However, there is also a significant group of points with low energy flux $\left(\sim 1-3 \mathrm{~mW} \mathrm{~m}^{-2}\right)$ which have a broad spread of mean electron energies in the range $\sim 50$ to $\sim 150 \mathrm{keV}$. These are generally associated with homogeneous and faint polar emission. Overall, the electron energy flux in the polar region is generally significantly lower than that in the main oval, while the mean energy of the precipitating electrons is comparable. Note that in some

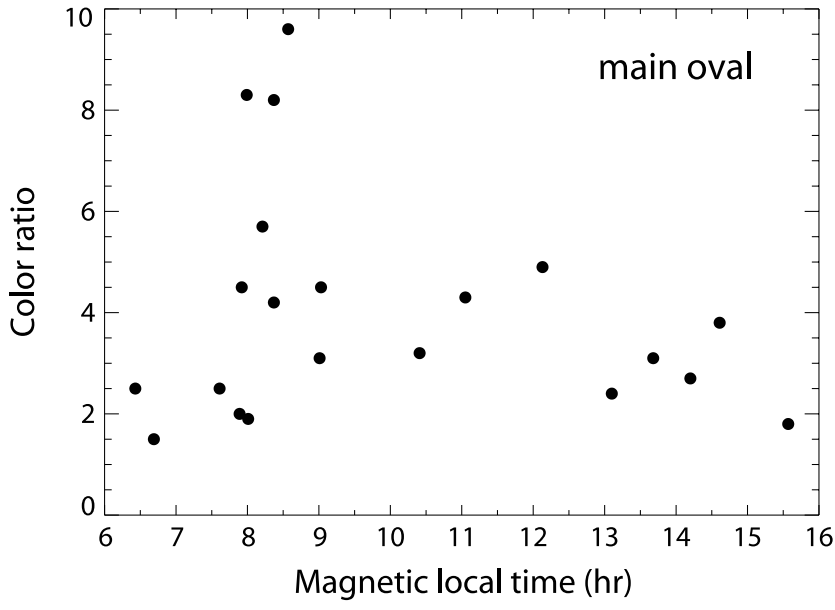

Figure 5. Relationship between the main oval CR and the magnetic local time derived from the mapped magnetic field lines.

cases, the count rate in the polar regions is very low compared with the bright main oval regions (for example the high-latitude regions in Figure 3c). Color ratios associated with these "dark polar regions," as identified by Grodent et al. [2003b], are thus noisier than in the main oval. To verify that emission from these dark regions originates from auroral processes with little contamination from the Jovian airglow, a spectrum of the high-latitude region was extracted for each case listed in Table 1. Each spectrum was examined, and it was confirmed that auroral processes were the dominant source of these emissions.

[21] The smaller values of the energy flux combined with the comparable mean electron energies noted above imply that the precipitating electron number flux and hence current density is lower in the polar regions than in the main oval. This is shown in Figure $4 d$ in the same format as Figure $4 b$. We note the same few points whose distribution is similar to that of the main oval, extending now from current densities of $\sim 0.05$ to $\sim 0.2 \mu \mathrm{A} \mathrm{m}^{-2}$ as the energy flux increases. There is also the other group of points with low energy flux whose current density is estimated to lie between $\sim 0.01$ and $0.03 \mu \mathrm{A} \mathrm{m}^{-2}$, i.e., an order of magnitude lower than that of the main oval.

\subsubsection{Color Ratio Variation With Magnetic Local Time}

[22] Positioning of the spectral slit relative to the auroral images allows us to determine the planetocentric latitudes and longitudes of the main oval segment intercepted by the STIS aperture. With these positions, it is possible to determine the magnetic local time (MLT) of the observations from the field line mapping to the equatorial plane, using the VIP4 magnetic field model [Connerney et al., 1998]. Figure 5 shows that no clear relationship is observed between the CR of the main oval spectra and the MLT so determined. However, the region close to 0800 MLT is associated with a wide range of color ratios (1.5 to 9.6) and thus with mean electron energies between 35 and $205 \mathrm{keV}$. This MLT region is associated with the morning storms that have been observed with HST in the imaging mode [Gérard et al., 1994; Clarke et al., 1998; Ballester et al., 1996; Grodent et al., 2003a]. The storms are characterized by a 

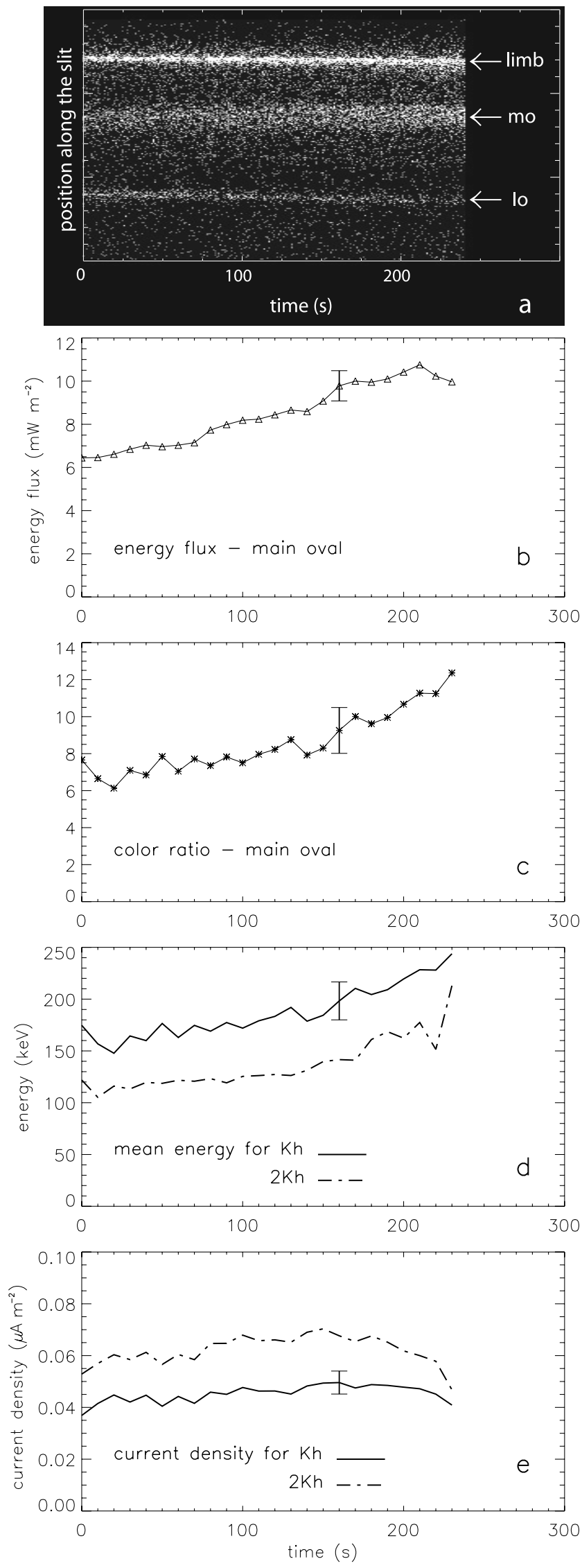

rapid (few $100 \mathrm{~s}$ ) increase in the brightness of the main oval near the dawn limb to values higher than those occurring elsewhere in the oval, followed by decreases in intensity. They are also found to remain fixed in local time over periods of up to several hours. It is believed that they are associated with the contraction of the dawn flank of the distant and middle magnetosphere as the planet rotates, leading to instabilities in the trapped corotating plasma. Our results show that the mean electron energy may exceed $200 \mathrm{keV}$ in this sector, implying that additional energy is provided to the electron population in comparison with the main oval at other MLTs.

\subsection{Time Variations}

\subsubsection{Global Analysis}

[23] All observations listed in Table 1 were obtained in the time-tagged mode, allowing variations of both the unabsorbed intensity and the CR to be examined for each interval. It is seen in column 10 of the table that during these intervals, the intensity does not show a significant change in 11 cases, changes by less than a factor of 2 in 10 cases, and exhibits significant changes in nine cases. These changes may represent either real time variations, or they may be caused by a brighter (or dimmer) segment of the oval moving into the slit aperture. Simulations made with STIS FUV images have been used to examine this space-time ambiguity. If the slit position is shifted dawnward by $\sim 2.5^{\circ}$, simulating the planetary rotation angle over $600 \mathrm{~s}$, it is found that the intensity may vary by a factor as large as 2 or 3. Consequently, the variations observed in the time-tagged spectra may be due to both longitudinal brightness gradients, as well as to intrinsic temporal changes. In the following discussion, the term "time variation" thus refers to changes observed in the STIS aperture during the time-tagged sequence, independent of whether they are due to temporal or spatial effects.

[24] Overall, the observations show that these variations are steady and continuous and do not show rapid bursts over $\sim 50-100 \mathrm{~s}$, which are typical of the high-latitude regions [Gérard et al., 2003]. It is also found that variations in the intensity are strongly correlated with variations in the CR (in eight cases) and that when the intensity remains constant, the CR also does not show significant variations.

Figure 6. Time variations observed in the spectral mode starting at 1647 UT on 22 February 2000 with the $52^{\prime \prime} \times$ $0.5^{\prime \prime}$ aperture (case 9 in Table 1). The figure panels show (a) the time-tagged sequence where each photon event on the MAMA detector in the unabsorbed spectral region above $1500 \AA$ is represented as an individual point, (b) the time variation of the electron energy flux averaged over $10 \mathrm{~s}$ segments and smoothed over 3 data points, (c) the corresponding $\mathrm{CR}$, which is strongly correlated with the energy flux, (d) the variation of the mean electron energy derived from the color ratio using an eddy diffusion coefficient $K_{h}=1.4 \times 10^{6} \mathrm{~cm}^{2} \mathrm{~s}^{-1}$ at the homopause (solid line) and twice this value (dot-dashed line), and (e) the precipitating electron current density obtained by dividing the energy flux by the mean energy and multiplying by the electron charge. 
These overall conclusions will now be illustrated with a few examples.

\subsubsection{Case Studies}

[25] In this section we analyze three typical cases of time variations of the main oval emissions. Figure 6 shows the time-tagged spectral sequence starting at 1647 UT on 22 February 2000 (case 9 in Table 1). The STIS slit crossed the Io trailing tail, the dayside main oval, the dayside high-latitude emission (described as the "dark region" by Grodent et al [2003b]), and the nightside main oval (limb). Figure $6 \mathrm{a}$ shows the $240 \mathrm{~s}$ spectral time sequence, where each photon event on the MAMA detector in the unabsorbed spectral region above $1500 \AA$ is represented as an individual point. The regions intercepted by the slit are indicated on the right-hand side of the plot. The Io tail shows a slight decrease in intensity during the exposure, while the limb shows a small increase. The main oval shows a somewhat diffuse emission because the slit, located close to the morning ansa of the oval, is influenced by limb brightening [Grodent et al., 1997]. Figures $6 \mathrm{~b}-6 \mathrm{~d}$ show the electron energy flux, the CR, and the mean electron energy for the main oval, the latter parameter being computed using standard (solid line) and increased (dot-dashed line) values of the eddy diffusion coefficient at the homopause, as indicated above. It can be seen that the energy flux (brightness) of the oval increases steadily over the interval, reaching a maximum of $11 \mathrm{~mW} \mathrm{~m}^{-2}(\sim 130 \mathrm{kR})$ near the end of the exposure. The $\mathrm{CR}$, initially close to 7 , is positively correlated with the intensity, and reaches a value of 12 at the end of the interval. These large CR values suggest high-energy electron precipitation, with mean energies increasing with the energy flux between $\sim 150$ and $\sim 250 \mathrm{keV}$, as seen in Figure 6d. Figure $6 \mathrm{f}$ shows the associated electron current density, obtained as above by dividing the energy flux by the mean energy (and multiplying by the charge). It can be seen that the correlated variations of these parameters are such that the electron current density (i.e., the electron number flux) remains nearly constant during the interval. The implied current density is $\sim 0.045 \mu \mathrm{A} \mathrm{m}^{-2}$ using the mean electron energies derived using the "standard" eddy diffusion coefficient (solid line), increasing to $\sim 0.065 \mu \mathrm{A} \mathrm{m}^{-2}$ if the eddy diffusion coefficient is doubled (dot-dashed line).

[26] In Figure 7 we similarly show a $230 \mathrm{~s}$ sequence from a time-tagged observation starting at 0614 UT on 14 November 2000 (case 11 in Table 1). It followed a $120 \mathrm{~s}$ image obtained $16 \mathrm{~min}$ before the spectrum, shown in Figure 3. The limb and the high-latitude regions intercepted by the slit do not exhibit appreciable time variation. As seen previously in Figure 3, however, the main oval forms two distinct regions of emission, termed "main ovals 1 and 2," which show distinct temporal behavior. It thus seems likely in this case that the temporal variation of the time-tagged spectra mainly reflect the rotational motion of the two subovals across the slit during the $230 \mathrm{~s}$ exposure. For main oval 1 at higher latitudes the energy flux increases steadily by a factor of more than two during the interval, from $\sim 18$ to $\sim 38 \mathrm{~mW} \mathrm{~m}^{-2}$, while the $\mathrm{CR}$ also exhibits a correlated increase, from initial values of $\sim 5$ to final values of $\sim 13$, implying an increase in mean electron energy from $\sim 120$ to $\sim 250 \mathrm{keV}$. This correlated behavior is thus similar to that observed in Figure 6. It again implies that the electron current density is approximately constant during the interval, $\sim 0.15 \mu \mathrm{A} \mathrm{m}^{-2}$ in this case $\left(\sim 0.2 \mu \mathrm{A} \mathrm{m}^{-2}\right.$ if the eddy diffusion coefficient is doubled). Over the same interval, main oval 2 at lower latitudes has nearly constant energy flux during the first $130 \mathrm{~s}$ and then declines during the last $100 \mathrm{~s}$. The energy flux in this emission is initially much higher than that of main oval 1 , while the CR is similar, suggesting precipitating electrons of $\sim 100 \mathrm{keV}$ in both cases. However, for main oval 2 this energy remains approximately constant as the intensity subsequently drops, thus illustrating a case where variations of the unabsorbed intensity and the CR are uncorrelated. The implication is that the electron current density is initially steady at a value of $\sim 0.3 \mu \mathrm{A} \mathrm{m}^{-2}$, twice as large as for main oval 1 , but subsequently declines to $\sim 0.2 \mu \mathrm{A} \mathrm{m}^{-2}$ as seen in Figure $7 \mathrm{i}$.

[27] A third case is illustrated in Figure 8. This longer $630 \mathrm{~s}$ sequence was obtained starting at $1234 \mathrm{UT}$ on 16 December 2000 (case 17 in Table 1) and followed a $100 \mathrm{~s}$ image obtained $8 \mathrm{~min}$ earlier (not shown). The STIS slit intercepted the limb, the high-latitude emissions, and, as in the case of Figure 7, a main oval that is divided into two distinct regions. The limb emission is fairly constant during the time interval, while in the high-latitude region the slit crosses the "swirl region," a region of patchy and shortlived (tens of seconds) faint emission [Grodent et al., 2003b]. These small and short-lived high-latitude emission features are well observed in Figure 8a. Figures $8 \mathrm{~b}-8 \mathrm{e}$ display the time variations of the emission parameters for main oval 1 . The energy flux associated with the emission, initially close to $25 \mathrm{~mW} \mathrm{~m}^{-2}$, drops rapidly during the first $30 \mathrm{~s}$ and then continues to decreases to reach a value of $2 \mathrm{~mW} \mathrm{~m}^{-2}$ at the end of the interval. The associated CR has a high value at the start of the exposure, $\sim 9$, and undergoes large variations during the first $100 \mathrm{~s}$ that show no apparent correlation with the unabsorbed intensity. After $100 \mathrm{~s}$, the $\mathrm{CR}$ and intensity undergo pronounced correlated decreases, with $\mathrm{CR}$ values of $\sim 2.5$ being reached at the end. This correlated behavior again implies an almost constant value of the electron current density after the initial drop, equal in this case to $\sim 0.05 \mu \mathrm{A} \mathrm{m}^{-2}$, as can be seen in Figure 8e. The upper panel of Figure 8 indicates the appearance of a second oval component after $400 \mathrm{~s}$, the parameters of which are shown in Figures $8 \mathrm{f}-8 \mathrm{i}$. The first $50 \mathrm{~s}$ in these panels show a rapid decrease, which is due to the presence of main oval 1. In order to catch the whole of main oval 2, the sum of the lines along the slit corresponding to main oval 2 includes two or three pixel lines that belong to main oval 1 . Thus disregarding the first $100 \mathrm{~s}$, which includes the broad extension of main oval 1, the energy flux associated with main oval 2 remains constant from 100 to $400 \mathrm{~s}$, then shows a brightening that attenuates after $600 \mathrm{~s}$. The CR of main oval 2 is seen to be remarkably correlated with the intensity, with values in the range of $\sim 2$ to $\sim 5$ (disregarding the first $100 \mathrm{~s}$ ). These values imply that the mean electron energy increases from $\sim 60$ to $\sim 130 \mathrm{keV}$ during the last $500 \mathrm{~s}$ as the auroral intensity also increases. Figure $8 \mathrm{i}$ then indicates that these variations imply an electron current density whose magnitude increases from $\sim 0.035$ to $\sim 0.05 \mu \mathrm{A} \mathrm{m}^{-2}$ concurrently with the brightening.

[28] Figures 6-8 illustrate some of the typical situations encountered in the database used in this study. Overall, examples can be found of correlation, lack of correlation, and anticorrelation, though anticorrelation is suspected in 

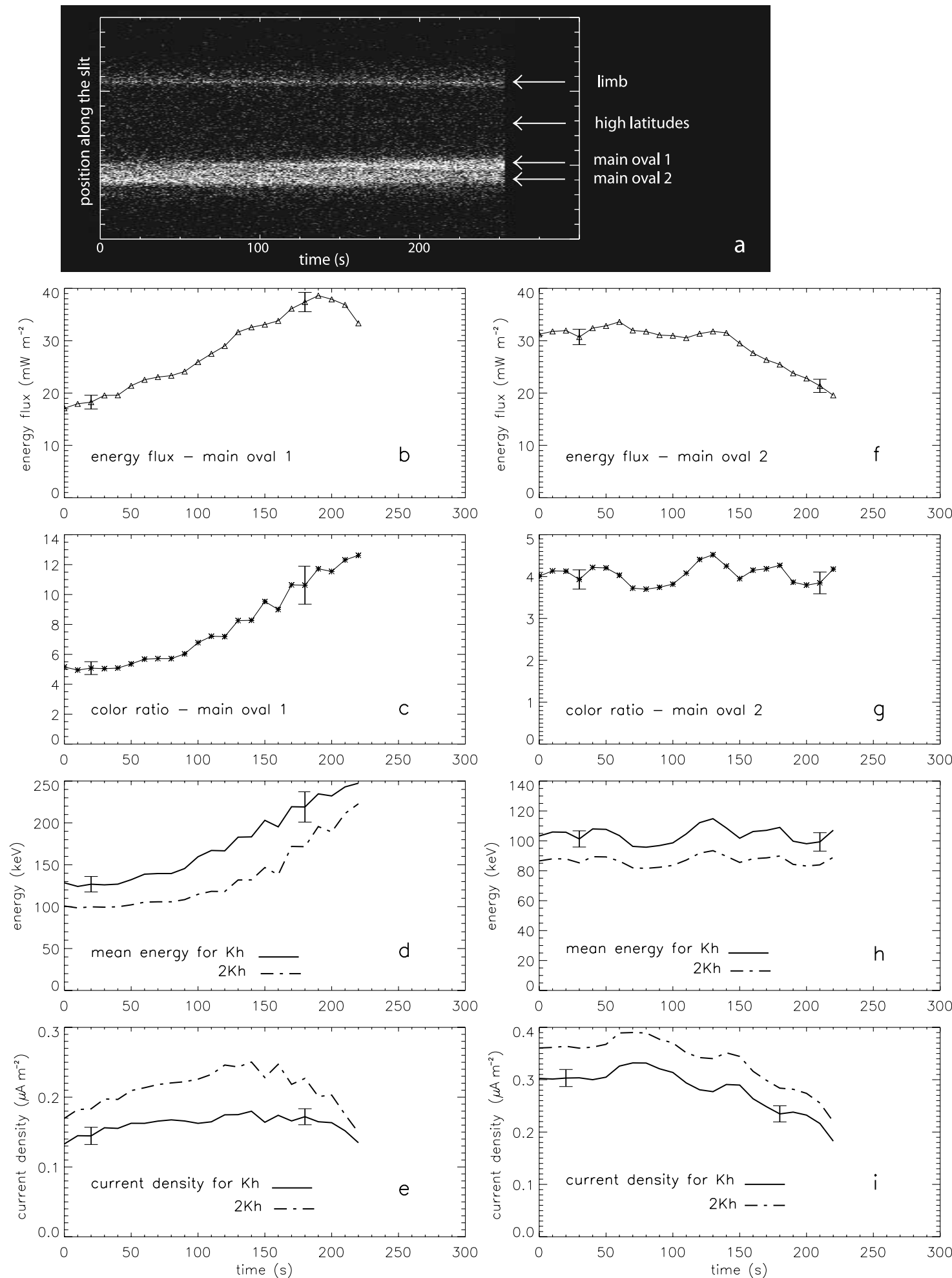

Figure 7. Time variations observed in the spectral mode starting at 0614 UT on 14 November 2000 with the $52^{\prime \prime} \times 0.5^{\prime \prime}$ aperture (case 11 in Table 1). (a)-(e) The same parameters for main oval 1 as in Figure 6. (f)-(i) The energy flux, CR, mean electron energy, and current density for main oval 2. In this case the energy flux remains approximately constant until $140 \mathrm{~s}$ and then decreases during the last $90 \mathrm{~s}$, while the CR does not exhibit a significant variation. This is a typical case where the energy flux and CR are uncorrelated. 

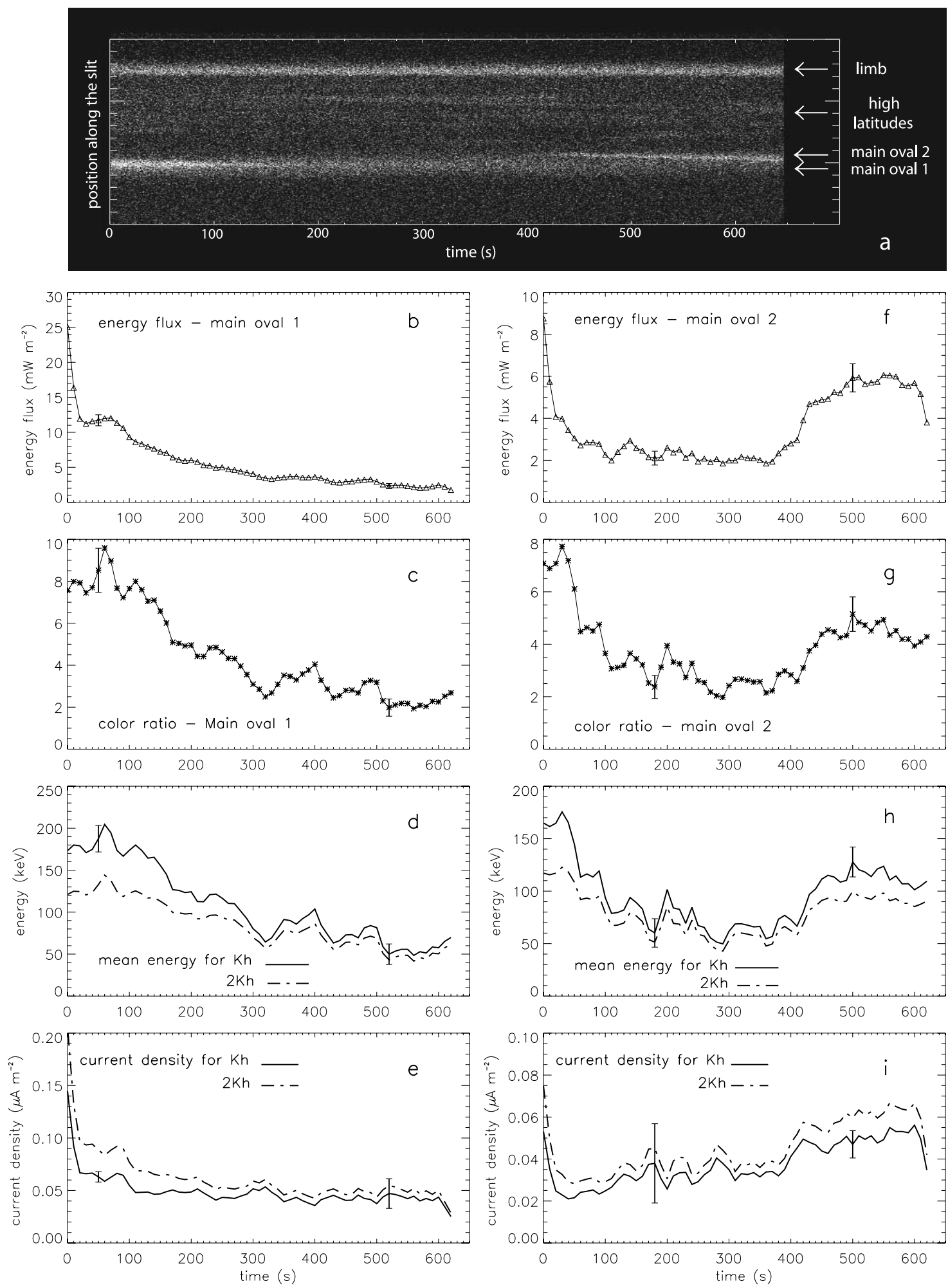

Figure 8. As for Figure 7 but for observations starting at 1234 UT on 16 December 2000 (case 17 in Table 1). In this case the two components of the oval are observed to vary independently of each other but with correlated variations of the $\mathrm{CR}$ in each case. 
one case only. On the other hand, many sequences do not show significant time variations and thus do not allow us to make a useful statement beyond this fact. When intensity changes occur, they are often positively correlated with the $\mathrm{CR}$, and thus with the electron mean energy, such that the electron current density remains relatively constant. However, examples are also present in which correlated changes in the current occur as well. In the next section we will consider these results in relation to existing theory and suggest possible interpretations.

\section{Discussion}

[29] The results described above, and elsewhere, clearly indicate that the three regions of Jovian auroral emission are associated with distinct magnetospheric plasma processes [Clarke et al., 1996]. The Io trailing tail extends downstream from the moon's magnetic footprint along the orbit, and a possible explanation is a plasma pickup from Io by the corotating Jovian magnetic field [Hill and Vasyliunas, 2002]. The polar emission is characterized by fast temporal variations [e.g., Grodent et al., 2003b], up to the extreme case of the polar flares described by Waite et al. [2001], where the brightness can increase by over an order of magnitude in a few tens of seconds. By contrast, the main oval emission is relatively constant in time [e.g., Grodent et al., 2003a], the time-tagged data shown here generally exhibiting only steady changes over intervals of several minutes. The results shown in Figure 4, however, indicate that the electrons producing both main oval and polar emissions are similar in energy, $\sim 50$ to $\sim 200 \mathrm{keV}$, but that the electron number and energy fluxes are usually larger for the main oval than for the polar region.

[30] We now consider the relation between the results obtained here and expectations derived from previous theoretical studies, focusing first on the main oval. As indicated in the introduction, theoretical discussion has associated the main oval with the corotation enforcement current circuit in the Jovian middle magnetosphere, specifically with the region of upward field-aligned current that flows in the inner part of the system [Bunce and Cowley, 2001; Cowley and Bunce, 2001; Hill, 2001; Saur et al., 2003]. We have already pointed out above that the magnitude of the currents derived here, as well, of course, as the "oval" morphology, are both consistent with theoretical estimates made on this basis [Cowley and Bunce, 2001; Cowley et al., 2002, 2003; Nichols and Cowley, 2004]. In these theoretical papers the current is calculated from consideration of the angular momentum transfer from the rotating atmosphere to the equatorial magnetospheric plasma. Additional considerations are required, however, to derive estimates of the precipitating electron energy flux and mean energy that can be compared with the results derived here. As also mentioned in the introduction, two such scenarios have been discussed to date. In the theoretical works by Cowley and Bunce [2001] and Cowley et al. $[2002,2003]$ it is shown that the upward field-aligned currents required by the angular momentum transfer considerably exceed those that can be carried unaided by precipitating hot magnetospheric electrons. They therefore propose that steady electric fields exist along the auroral field lines that accelerate magnetospheric electrons toward the ionosphere, and they calculate the parameters of the resulting precipitation from the kinetic theory due to Knight [1973]. Alternatively, Saur et al. [2003] have suggested that the auroral electrons are heated and accelerated by an electric field established by an "anomalous turbulent resistivity" associated with the dissipation of Alfvén wave energy radiated from the plasma sheet. Here we will consider the compatibility of the predictions of these scenarios with the results derived above. We start with the "Knight" picture, in which the field-aligned current is associated with a steady field-aligned voltage, and begin by briefly reviewing the relevant theory.

[31] The maximum value of the upward-directed ionospheric current density that can be carried by precipitating magnetospheric electrons without field-aligned acceleration is

$$
j_{\| i 0}=e N\left(\frac{W_{t h}}{2 \pi m_{e}}\right)^{1 / 2},
$$

where $e$ and $m_{e}$ are the charge and mass of the electron, and $N$ and $W_{t h}$ are the number density and thermal energy of the "source" magnetospheric electron population, assumed to have an isotropic Maxwellian distribution, such that $W_{t h}=$ $k_{B} T$. This limiting current density corresponds to the case of a full down-going loss-cone and an empty up-going loss cone at the top of ionosphere. In order produce a current density $j_{\| i}$ larger than $j_{\| i 0}$, as will generally be the case in the Jovian middle-magnetosphere coupling current system, the field-aligned voltage required is

$$
e \Phi_{\|}=W_{t h}\left[\left(\frac{j_{\| i}}{j_{\| i 0}}\right)-1\right],
$$

where it has been assumed that the acceleration region is sufficiently compact that no particle mirrors before experiencing the full voltage drop and that the "top" of the voltage drop occurs well above the minimum height given approximately by $\left(r_{\min } / R_{J}\right) \approx\left(j_{\| i} / j_{\| i 0}\right)^{1 / 3}$ (see Cowley and Bunce [2001] for further details). The latter expression in turn assumes a dominant dipole planetary field in the polar regions. The enhanced precipitating electron energy flux that corresponds to equation (2) is then

$$
E_{f i}=\frac{E_{f i 0}}{2}\left[\left(\frac{j_{\| i}}{j_{\| i 0}}\right)^{2}+1\right] \text {, }
$$

where $E_{f i 0}$ is the field-aligned energy flux for no fieldaligned acceleration

$$
E_{f i 0}=2 N W_{t h}\left(\frac{W_{t h}}{2 \pi m_{e}}\right)^{1 / 2}
$$

obtained with the same assumptions as for equation (1). These are the forms of the equations employed in the theoretical studies of Cowley and Bunce [2001] and Cowley et al. [2002, 2003], where voltages and energy fluxes were derived from calculated field-aligned current densities.

[32] Equation (3) provides a relation between the electron current and electron energy flux, which, for given magne- 
tospheric electron "source" parameters, can be compared directly with the experimental current-energy flux values shown in Figure $4 \mathrm{~b}$. This equation can also be inverted to give

$$
\left(\frac{j_{\| i}}{j_{\| i 0}}\right)=\left(2\left(\frac{E_{f i}}{E_{f i 0}}\right)-1\right)^{1 / 2}
$$

from which the mean energy of the precipitating electrons $\langle W\rangle$ can be obtained in terms of the energy flux from the ratio of the energy flux and the number flux $\left(F_{\| i}=j_{\| i} / e\right)$

$$
\langle W\rangle=2 W_{t h} \frac{\left(\frac{E_{f i}}{E_{f i 0}}\right)}{\left(2\left(\frac{E_{f i}}{E_{f i 0}}\right)-1\right)^{1 / 2}} .
$$

This expression can then be compared with the experimental results shown in Figure $4 \mathrm{a}$. We note, however, that the usual regime of interest is one in which $j_{\| i} \gg j_{\| i 0}$, $E_{f i} \gg E_{f i 0}$, and hence $\langle W\rangle \gg W_{t h}$, in which case we find

$$
\begin{aligned}
& j_{\| i} \approx \sqrt{2} j_{\| i 0}\left(\frac{E_{f i}}{E_{f i 0}}\right)^{1 / 2} \propto \frac{N^{1 / 2}}{W_{t h}^{1 / 4}} E_{f i}^{1 / 2}, \\
& \langle W\rangle \approx \sqrt{2} W_{t h}\left(\frac{E_{f i}}{E_{f i 0}}\right)^{1 / 2} \propto \frac{W_{t h}^{1 / 4}}{N^{1 / 2}} E_{f i}^{1 / 2},
\end{aligned}
$$

and $\langle W\rangle \approx e \Phi_{\|}$. In this limit therefore we find that both the current density and the mean electron energy should increase as the square root of the energy flux. We also find that at a given energy flux $E_{f i} \gg E_{f i 0}$, the average energy depends on the parameters of the source electron population in proportion to the fourth root of the thermal energy $W_{\text {th }}$ and inversely as the square root of the number density $N$. The field-aligned voltage scales with the source electron parameters in the same way, while the associated field-aligned current density scales inversely as this ratio.

[33] In the above theoretical studies the magnetospheric "source" electrons have been taken to be the "warm" population observed by during the Voyager fly-bys, which envelopes the middle magnetosphere plasma sheet and extends outside it to higher latitudes [Scudder et al., 1981]. The Voyager observations show that this population has temperatures of $2-3 \mathrm{keV}$, and densities of $0.001-$ $0.01 \mathrm{~cm}^{-3}$. This compares with values of several tens of $\mathrm{eV}$ and densities of $\sim 0.1-10 \mathrm{~cm}^{-3}$ for the cold thermal electrons which are electrostatically trapped with the outflowing heavy ions within the equatorial middle magnetosphere plasma sheet. In the above theoretical work, the parameters employed were $W_{t h}=2.5 \mathrm{keV}$, corresponding to the center of the energy range, and $N=0.01 \mathrm{~cm}^{-3}$, corresponding to the upper value of the density. The resulting relationships between the mean energy of the precipitating electrons and the electron energy flux obtained from equation (6), and the current density and the energy flux obtained from equation (5), are shown by the dotdashed lines in Figures $4 \mathrm{a}$ and $4 \mathrm{~b}$. It can be seen that the overall trend of these curves is similar to those derived from the HST-STIS data but that they pass close only to the lowermost points in the mean energy plot and to the uppermost points of the current plot. If, however, we employ the same source electron thermal energy, i.e., $2.5 \mathrm{keV}$, but reduce the number density to the lower end of the range given by Scudder et al. [1981], i.e., to $0.001 \mathrm{~cm}^{-3}$, then we obtain the dashed curves shown in Figures $4 \mathrm{a}$ and $4 \mathrm{~b}$, which now pass close to the uppermost points in Figure $4 \mathrm{a}$ and the lowermost in Figure $4 \mathrm{~b}$. We thus find that employing $W_{t h}=$ $2.5 \mathrm{keV}$ (the center energy of the narrow range quoted by Scudder et al. [1981]) and the full range of densities $0.001-0.01 \mathrm{~cm}^{-3}$ essentially brackets the data points. A least-squares fit to the data shown in Figure $4 \mathrm{a}$ of the theoretical line given by equation (6) with $W_{t h}=2.5 \mathrm{keV}$ yields a best fit for a source density of $N \approx 0.0026$, shown by the central solid line in the figure. The corresponding line also passes centrally through the current density values shown in Figure 4b. Overall, these results demonstrate a clear compatibility between the experimental data and the theoretical formulation based on Knight's [1973] kinetic theory of field-aligned currents. We note, however, that similar fits to the data could also be obtained with differing electron source parameters which maintain similar values of $W_{t h}^{1 / 4} / N^{1 / 2}$ (see equations (7) and (8)).

[34] The above theoretical considerations also provide an interpretation of the main oval time-tagged results shown in Figures 6-8. One result shown in these figures is that when the main oval emission observed in the HST-STIS slit changes steadily with time, a correlated change often also takes place in the mean electron energy, such that the current density (electron number flux) remains approximately constant. Within the above theoretical framework, this behavior implies the existence of a near-steady magnetosphere-ionosphere coupling current system, but where the parameters of the "warm" magnetospheric source electrons change slowly with time in the region observed, such that the auroral parameters also change slowly with time. For example, the intensity increase observed in Figure 6 and in main oval 1 in Figure 7 could be caused by an increase in the thermal energy of the source electrons, a decrease in the number density, or both (see equations (5) and (7)), and vice versa for the intensity decrease observed in main oval 1 in Figure 8. In principle we cannot tell from these measurements whether it is the source thermal energy or the density which is responsible (or both), but a variation in the density seems the most probable, considering both the Voyager observations presented by Scudder et al. [1981] and the fact that at fixed current density the precipitating energy flux varies somewhat insensitively as the square root of the temperature but inversely with the density (equation (7)). Assuming a fixed thermal energy $W_{t h}=2.5 \mathrm{keV}$, we find using equation (3) (or equation (5)) that the increase in the main oval intensity observed in Figure 6 is consistent with a decrease in the source density from $\sim 0.0006 \mathrm{~cm}^{-3}$ at the beginning of the interval to $\sim 0.0004 \mathrm{~cm}^{-3}$ at the end. (For a different choice of thermal energy the derived densities would vary in proportion to the square root of the thermal energy, so the values are not very sensitive to this choice.) Similarly, the intensity increase observed in main oval 1 in Figure 7 is consistent with a source density decrease from $\sim 0.002$ to $\sim 0.001 \mathrm{~cm}^{-3}$, while the intensity decrease observed in main oval 1 in Figure 8 (after the initial step) 
is consistent with a source density increase from $\sim 0.0006$ to $\sim 0.002 \mathrm{~cm}^{-3}$. Moderate density changes of this nature in the region observed during the measurements are perhaps not implausible. On the other hand, the variation in main oval 2 in Figure 7 does imply a change in the current density as seen in the figure, as well (possibly) as in the source electron parameters. The current density changes from $\sim 0.3 \mu \mathrm{A} \mathrm{m}^{-2}$ at the beginning of the interval to $\sim 0.2 \mu \mathrm{A} \mathrm{m}^{-2}$ at the end, as can be seen in Figure $7 \mathrm{i}$, while assuming a fixed source thermal energy of $2.5 \mathrm{keV}$ as above, the inferred change in magnetospheric source density over the interval obtained from equations (3) or (5) is from $\sim 0.005 \mathrm{~cm}^{-3}$ at the beginning to $\sim 0.004 \mathrm{~cm}^{-3}$ at the end. Similarly for main oval 2 in Figure 8, the current density increases from $\sim 0.03$ to $0.05 \mu \mathrm{A} \mathrm{m}^{-2}$ during the last $500 \mathrm{~s}$ of the observation, while the inferred source density changes from $\sim 0.001$ to $\sim 0.0008 \mathrm{~cm}^{-3}$. In these cases therefore the observed variations in intensity are inferred to have been caused more by changes in the current density in the region observed, with relatively fixed source plasma parameters.

[35] As indicated above, an alternative scenario for the formation of field-aligned voltages in the magnetosphereionosphere current circuit associated with the main oval has been proposed by Saur et al. [2003], which we now also discuss in relation to our data. In this picture, weak MHD turbulence observed within the middle magnetosphere plasma sheet radiates Alfvén waves along the field lines toward the ionosphere, where their energy is partially dissipated to electron heating and acceleration from the observed wave amplitudes, as the cross-field spatial scales become short in the increasing field strength. Weak turbulence theory is used to estimate the energy flux dissipated to electron heating from the observed wave amplitudes, this amounting to $\sim 20 \%$ of the observed Alfvén wave energy flux. The observations indicate that the energy flux dissipated to electron peaks at $3.2 \times 10^{-7}$, at a radial distance of $\sim 20 \mathrm{R}_{\mathrm{J}}$ in the near-equatorial magnetosphere. Assuming that all of this energy appears as auroral electrons at the feet of the field lines, the energy flux input to the main oval is estimated to be $\sim 7.4 \mathrm{~mW} \mathrm{~m}^{-2}$. The latter value has been obtained by multiplying the equatorial energy flux quoted above by the ratio of the background magnetic field strengths, where the field strength in the magnetosphere is the value corresponding to the fluctuation data set, quoted as 43 nT by Saur et al. [2003], and the field strength in the polar ionosphere is taken to be $\sim 10^{6} \mathrm{nT}$. To determine the value of the accelerating voltage, and hence the energy of the precipitating electrons, Saur et al. [2003] then divide the equatorial energy flux quoted above by the corresponding equatorial field-aligned current density, taken to be $2 \times$ $10^{-12}$ on the basis of the results presented by Khurana [2001], to determine a voltage of $160 \mathrm{kV}$. The related current density at ionospheric heights is $\sim 0.05 \mu \mathrm{A} \mathrm{m}{ }^{-2}$. The resulting estimates (i.e., a precipitating energy flux of $7.4 \mathrm{~mW} \mathrm{~m}^{-2}$ associated with an electron energy of $160 \mathrm{keV}$ and a current density of $\sim 0.05 \mu \mathrm{A} \mathrm{m}^{-2}$ ) are shown by the large dots in Figures $4 \mathrm{a}$ and $4 \mathrm{~b}$. These values are not dissimilar to those obtained here from the HST-STIS data, but the energy flux lies on the low side of the experimental values, while the electron energy lies on the high side of these values. However, reasons exist to suppose that the magnetospheric field strength used to perform the above energy flux mapping to the ionosphere may be too high, such that the resulting energy flux value is too low (J. Saur, personal communication, 2003). In addition, we would also comment that the near-equatorial field-aligned current density assumed in the calculation also yields a value of the ionospheric current density which seems rather low for the peak of the field-aligned current density in the main oval, by factors of at least two. Use of a somewhat higher current density appropriate to conditions near the peak would yield smaller estimates of the accelerating voltage, bringing the estimate nearer to values derived experimentally here.

[36] Turning finally to the polar emissions, results for which are shown in Figures $4 \mathrm{c}-4 \mathrm{~d}$, it has already been pointed out that the data may be roughly divided into two groups. The first is associated with relatively high values of the current density, $\sim 0.1$ to $\sim 0.2 \mu \mathrm{A} \mathrm{m}^{-2}$, and precipitating energy flux, $\sim 5$ to $\sim 20 \mathrm{~mW} \mathrm{~m}^{-2}$, which are not dissimilar to values in the main oval. The corresponding mean electron energies are $\sim 50$ to $\sim 100 \mathrm{keV}$. The other group, which contains most of the data points, is associated with low values of the energy flux $\sim 1$ to $\sim 3 \mathrm{~mW} \mathrm{~m}^{-2}$ but with comparable mean electron energies, $\sim 50$ to $\sim 150 \mathrm{keV}$, such that the electron current density is much lower, $\sim 0.01$ to $\sim 0.03 \mu \mathrm{A} \mathrm{m} \mathrm{m}^{-2}$. It seems inescapable that the first of these groups of points, at the least, are, like the main oval, associated with an auroral acceleration mechanism, as opposed, for example, to being due to direct precipitation from a quasi-isotropic hot magnetospheric electron source. In the latter case we can easily estimate the implied pressure of the magnetospheric electrons from the precipitating energy flux and the mean electron energy, using equations (1) and (4). For the first group of points the resulting values lie in the range $\sim 0.1$ to $\sim 0.5 \mathrm{nPa}$, which, even setting aside the pressure contribution of corresponding magnetospheric ions, already considerably exceed the field pressures in the outer magnetosphere and Jovian tail to which the polar regions presumably map. Field strengths in these regions of $\sim 2$ to $10 \mathrm{nT}$ correspond to magnetic pressures of $\sim 0.002$ to $\sim 0.04 \mathrm{nPa}$, roughly an order of magnitude less. We thus infer that the bright polar emissions $(\sim 50$ to $\sim 200 \mathrm{kR})$ associated with such elevated electron energy fluxes must involve auroral acceleration from a lower-energy magnetospheric source, possibly via the same physical mechanism that produces the main oval. For the second group of polar points at lower energy flux, however, the situation is less clear-cut. The electron pressures deduced from these data are $\sim 0.05 \mathrm{nPa}$, equivalent to the pressure of a magnetic field of $\sim 12$ nT. Precipitation from a quasi-isotropic hot outer magnetospheric source is thus not clearly ruled out on this basis.

\section{Summary}

[37] Low-resolution HST-STIS spectra of the Jovian aurora have been obtained and used to study the relationship between the energy flux and the mean energy of precipitating electrons at the top of the Jovian atmosphere. The electron energy fluxes are deduced from the auroral brightness, while the mean energies are deduced from the color ratio, which is a measure of the attenuation of the $\mathrm{H}_{2}$ emission by hydrocarbons (mainly methane). It has been 
shown that for the main oval, the mean electron energy (lying between $\sim 30$ and $\sim 200 \mathrm{keV}$ ) is positively correlated with the energy flux $\left(\sim 2\right.$ to $\left.\sim 30 \mathrm{~mW} \mathrm{~m}^{-2}\right)$, though with considerable scatter about typical values. No systematic dependence is found between the electron energy and magnetic local time, but the morning sector of the oval around 0800 MLT shows greater variability than other regions, possibly due to the occurrence of dawn storms. These data have also been used to compute the ionospheric current density carried by the main oval electrons, which is also found to be positively correlated with the electron energy flux. The values of the current density so determined $\left(\sim 0.04\right.$ to $\left.\sim 0.4 \mu \mathrm{A} \mathrm{m} \mathrm{m}^{-2}\right)$ are compatible with those expected on the basis of previous analyses of magnetospheric magnetic field data and from theoretical models of the middle magnetosphere angular momentum coupling current system. Time-tagged data have been used to show that when variations of the main oval emission occur, they are usually slow and steady over the few-minute intervals of observation and often involve correlated changes in the intensity and mean electron energy associated with nearconstant values of the electron current density. Examples are also found, however, where related changes in the current density also occur. All the observations we examined suggest that significant time variations can be due to (1) a change of the source parameters with the current held constant, (2) a variation of the current with the source parameters held fixed, or (3) a contribution of both phenomena. Although the cases presented here allowed to discriminate between these three origins of variation and to estimate their contribution in each case, the small number of observations with substantial time variations did not allow to make a global statement of the importance of each origin that would be statistically significant.

[38] Corresponding analyses of auroral emissions in the polar region indicate the possible existence of two populations. The first has characteristics similar to those of the main oval, though with typically somewhat smaller values of the energy flux and current density. The second is associated with similarly high-energy electrons ( $\sim 50$ to $\sim 150 \mathrm{keV}$ ) but with much smaller values of the energy flux $\left(\sim 2 \mathrm{~mW} \mathrm{~m}^{-2}\right)$ and current density $\left(\sim 0.02 \mu \mathrm{A} \mathrm{m}^{-2}\right)$.

[39] The results for the main oval have been compared with theoretical expectations based on Knight's [1973] kinetic theory of field-aligned electric field acceleration associated with field-aligned currents and with Saur et al.'s [2003] analysis of auroral electron heating associated with the dissipation of MHD turbulent energy. It has been shown that the experimental results are compatible with Knight's theory as implemented in the recent Jovian coupling current system analyses of Cowley and Bunce [2001] and Cowley et al. [2002, 2003], specifically with regard to the observed correlations between the mean electron energy, the current density, and the electron energy flux. Results are compatible with a magnetospheric source electron population with a thermal energy of $\sim 2.5 \mathrm{keV}$ and with a density varying between $\sim 0.001$ and $\sim 0.01 \mathrm{~cm}^{-3}$, consistent with Voyager "warm" magnetospheric electron observations presented by Scudder et al. [1981]. In terms of this theory the time-tagged results are interpreted as often being due to a near-steady magnetosphere-ionosphere coupling current system, combined with slow moderate variations in the magnetospheric "source" electron parameters. On the other hand, examples also occur where the auroral intensity variations appear mainly to be due to changes in the current density with relatively fixed source electron parameters. The physical picture presented by Saur et al. [2003] has not been developed to date to the point where similarly detailed comparisons can be made. However, the spot parameter values estimated by these authors have been found to be roughly comparable with those derived experimentally here.

[40] With regard to the polar emissions, arguments based on pressure balance have been advanced to show that the data associated with larger energy fluxes must also result from an auroral acceleration process, perhaps similar to that which results in the main oval emission. However, it remains possible that the emissions with low energy fluxes could also result from precipitation from a quasi-isotropic hot magnetospheric electron source.

[41] Acknowledgments. This work is based on observations with the NASA/ESA Hubble Space Telescope, obtained at the Space Telescope Science Institute (STScI), which is operated by the AURA, Inc. for NASA. JCG and DG acknowledge support from the Belgian Fund for Scientific Research (FNRS). The PRODEX program of ESA provided financial support for this research to the University of Liège. This research was also funded by grants from the Space Telescope Science Institute and from NASA to the University of Michigan. SWHC was supported by PPARC Senior Fellowship PPA/N/S/2000/00197.

[42] Arthur Richmond thanks the reviewers for their assistance in evaluating this paper.

\section{References}

Ballester, G. E., et al. (1996), Time-resolved observations of Jupiter's farultraviolet aurora, Science, 274, 409.

Barbosa, D. D., F. L. Scarf, W. L. Kurth, and D. A. Gurnett (1981), Broadband electrostatic noise and field-aligned currents in Jupiter's middle magnetosphere, J. Geophys. Res., 86, 8357

Bhattacharya, B., R. H. Thorne, and D. J. Williams (2001), On the energy source for diffuse Jovian auroral emissity, Geophys. Res. Lett., 28, 2751.

Bunce, E. J., and S. W. H. Cowley (2001), Divergence of the equatorial current in the dawn sector of Jupiter's magnetosphere: analysis of Pioneer and Voyager magnetic field data, Planet. Space Sci., 49, 1089.

Clarke, J. T., et al. (1996), Far-UV imaging of Jupiter's aurora with HST/ WFPC 2, Science, 274, 404.

Clarke, J. T., et al. (1998), Hubble Space Telescope imaging of Jupiter's UV aurora during the Galileo orbiter mission, J. Geophys. Res., 103, 20,217.

Clarke, J. T., et al. (2002), Ultraviolet auroral emissions from the magnetic footprints of Io, Ganymede, and Europa on Jupiter, Nature, 415, 997.

Clarke, J. T., et al. (2004), Jupiter's aurora, in Jupiter: The Planet, Satellites, and Magnetosphere, edited by F. Bagenal et al., Cambridge Univ. Press, New York, in press.

Connerney, J. E. P., M. H. Acuna, N. F. Ness, and T. Satoh (1998), New models of Jupiter's magnetic field constrained by the Io flux tube footprint, J. Geophys. Res., 103, 11,929.

Cowley, S. W. H., and E. J. Bunce (2001), Origin of the main auroral oval in Jupiter's coupled magnetosphere-ionosphere system, Planet. Space Sci., 49, 1067.

Cowley, S. W. H., et al. (1996), Plasma flow in the Jovian magnetosphere and related magnetic effects: Ullysses observations, J. Geophys. Res., $101,15,197$

Cowley, S. W. H., J. D. Nichols, and E. J. Bunce (2002), Distributions of current and auroral precipitation in Jupiter's middle magnetosphere computed from steady-state Hill-Pontius angular velocity profiles: Solutions for current sheet and dipole magnetic field models, Planet. Space Sci., 50, 717.

Cowley, S. W. H., E. J. Bunce, and J. D. Nichols (2003), Origins of Jupiter's main oval auroral emissions, J. Geophys. Res., 108(A4), 8002, doi:10.1029/2002JA009329.

Frank, L. A., and W. R. Paterson (2002), Galileo observations of electron beams and thermal ions in Jupiter's magnetosphere and their relationship to the aurora, J. Geophys. Res., 107(A12), 1478, doi:10.1029/ 2001JA009150.

Gehrels, N., and E. C. Stone (1983), Energetic oxygen and sulfur ions in the Jovian magnetosphere and their contribution to the auroral excitation, J. Geophys. Res., 88, 5537. 
Gérard, J.-C., and V. Singh (1982), A model of energetic electrons and EUV emission in the Jovian and Saturnian atmospheres and implications, J. Geophys. Res., 87, 4525

Gérard, J.-C., V. Dols, R. Prangé, and F. Paresce (1994), The morphology of the north Jovian ultraviolet aurora observed with the Hubble Space Telescope, Planet. Space Sci., 42, 905

Gérard, J.-C., J. Gustin, D. Grodent, P. Delamere, and J. T. Clarke (2002) The excitation of the FUV Io tail on Jupiter: Characterization of the electron precipitation, J. Geophys. Res., 107(A11), 1394, doi:10.1029/ 2002JA009410.

Gérard, J.-C., J. Gustin, D. Grodent, and J. T. Clarke (2003), Spectra observations of transient features in the FUV Jovian polar aurora J. Geophys. Res., 108(A8), 1319, doi:10.1029/2003JA009901.

Gladstone, G. R., M. Allen, and Y. L. Yung (1996), Hydrocarbon photochemistry in the upper atmosphere of Jupiter, Icarus, 119, 1.

Grodent, D., G. R. Gladstone, J.-C. Gérard, V. Dols, and J. H. Waite (1997) Simulation of the morphology of the Jovian UV north aurora observed with the Hubble Space Telescope, Icarus, 128, 306.

Grodent, D., J. H. Waite Jr., and J.-C. Gérard (2001), A self-consistent model of the Jovian auroral thermal structure, J. Geophys. Res., 106, 12,933.

Grodent, D., J. T. Clarke, J. H. Waite, J. Kim, and S. W. H. Cowley (2003a), Jupiter's main auroral oval observed with HST-STIS, J. Geophys. Res. 108(A11), 1389, doi:10.1029/2003JA009921.

Grodent, D., J. T. Clarke, J. H. Waite, S. W. H. Cowley, J.-C. Gérard, and J. Kim (2003b), Jupiter's polar auroral emissions, J. Geophys. Res. 108(A10), 1366, doi:10.1029/2003JA010017.

Gustin, J., D. Grodent, J.-C. Gérard, and J. T. Clarke (2002), Spatially resolved far ultraviolet spectroscopy of the Jovian aurora, Icarus, 157, 91.

Hill, T. W. (1979), Inertial limit on corotation, J. Geophys. Res., 84, 6554.

Hill, T. W. (1980), Corotation lag in Jupiter's magnetosphere: Comparison of observation and theory, Science, 207, 301.

Hill, T. W. (2001), The Jovian auroral oval, J. Geophys. Res., 106, 8101.

Hill, T. W., and V. M. Vasyliunas (2002), Jovian auroral signature of Io's corotational wake, J. Geophys. Res., 107(A12), 1464, doi:10.1029/ 2002JA009514

Isbell, J., A. J. Dessler, and J. H. Waite Jr. (1984), Magnetospheric energisation by interaction between planetary spin and the solar wind, J. Geophys. Res., 89, 10,716.

Kennel, C. F., and F. V. Coroniti (1975), Is Jupiter's magnetosphere like pulsar's or Earth's?, in The Magnetospheres of Earth and Jupiter, edited by V. Formisano, p. 451, D. Reidel, Norwell, Mass.

Khurana, K. K. (2001), Influence of solar wind on Jupiter's magnetosphere deduced from currents in the equatorial plane, J. Geophys. Res., 106, 25,999
Knight, S. (1973), Parallel electric fields, Planet. Space Sci., 21, 741.

Krupp, N., E. Keppler, R. Seidel, J. Woch, A. Korth, A. F. Cheng, S. E. Hawkins III, L. J. Lanzerotti, C. G. Maclennan, and M. K. Dougherty (1997), Field-aligned particle streaming in the duskside high latitude Jovian magnetosphere, Adv. Space Res., 20, 225.

Landsman, W. (1998), Characteristics of the FUV-MAMA dark rate, STSCI Rep. 57, Space Telesc. Sci. Inst., Baltimore, Md. (Available as http:// hires.gsfc.nasa.gov/stis/postcal/quick_reports/r057/r057.html)

Livengood, T. A., D. F. Strobel, and H. W. Moos (1990), Long-term study of longitudinal dependence in primary particle precipitation in the north Jovian aurora, J. Geophys. Res., 95, 10,375.

Mauk, B. H., B. J. Anderson, and R. M. Thorne (2002), Magnetosphereionosphere coupling at Earth, Jupiter, and beyond, in Atmosphere in the Solar System: Comparative Aeronomy, edited by M. Mendillo, A. Nagy, and J. H. Waite, pp. 97-114, AGU, Washington, D.C.

Nichols, J. D., and S. W. H. Cowley (2004), Magnetosphere-ionosphere coupling currents in Jupiter's middle magnetosphere: Effect of precipitation-induced enhancement of the ionospheric Pedersen conductivity, Ann. Geophys., 22, 1799-1827.

Saur, J., A. Pouquet, and W. H. Matthaeus (2003), An acceleration mechanism for the generation of the main auroral oval on Jupiter, Geophys. Res. Lett., 30(5), 1260, doi:10.1029/2002GL015761.

Scudder, J. D., E. C. Sittler Jr., and H. S. Bridge (1981), A survey of the plasma electron environment of Jupiter: A view from Voyager, J. Geophys. Res., 86, 8157.

Waite, J. H., Jr., T. E. Cravens, J. U. Kozyra, A. F. Nagy, S. K. Atreya, and R. H. Chen (1983), Electron precipitation and related aeronomy of the Jovian thermosphere and ionosphere, J. Geophys. Res., 88, 6143.

Waite, J. H., Jr., et al. (2001), An auroral flare at Jupiter, Nature, 410, 787

Yung, Y. L., G. R. Gladstone, K. M. Chang, J. M. Ajello, and S. K. Srivastava (1982), $\mathrm{H}_{2}$ fluorescence spectrum from 1200 to $1700 \AA$ by electron impact: Laboratory study and application to Jovian aurora, Astrophys. J., 254, L65-69.

J. T. Clarke, Center for Space Physics, Boston University, Boston, MA 02215, USA.

S. W. H. Cowley, Department of Physics and Astronomy, Leicester University, Leicester LE1 7RH, UK

J.-C. Gérard, A. Grard, D. Grodent, and J. Gustin, Laboratoire de Physique Atmosphérique et Planetaire, Université de Liège, Liège, Belgium. (jc.gerard@ulg.ac.be; gustin@astro.ulg.ac.be) 